\title{
Combinatorics of free vertex algebras
}

\author{
Michael Roitman \\ M.S.R.I. \\ 1000 Centennial Dr. \\ Berkeley, CA 94720 \\ E-mail: roitman@msri.org
}

Key Words: Free vertex algebras, lattice vertex algebras, conformal algebras

\section{INTRODUCTION}

This paper illustrates the combinatorial approach to vertex algebra study of vertex algebras presented by generators and relations. A necessary ingredient of this method is the notion of free vertex algebra. Borcherds [2] was the first to note that free vertex algebras do not exist in general. The reason for this is that vertex algebras do not form a variety of algebras, as defined in e.g. 泊, because the locality axiom (see $\$ 2$ below) is not an identity. However, a certain subcategory of vertex algebras, obtained by restricting the order of locality of generators, has a universal object, which we call the free vertex algebra corresponding to the given locality bound. In [15] some free vertex algebras were constructed and in certain special cases their linear bases were found. In this paper we generalize the construction of 115 and find linear bases of an arbitrary free vertex algebra.

It turns out that free vertex algebras are closely related to the vertex algebras corresponding to integer lattices. The latter algebras play a very important role in different areas of mathematics and physics. They were extensively studied in e.g. [5, 6, 9, 11, 14]. Here we explore the relation between free vertex algebras and lattice vertex algebras in much detail. These results comply with the use of the word "free" in physical literature refering to some elements of lattice vertex algebras, like in "free field", "free bozon" or "free fermion".

Among other things, we find a nice presentation of lattice vertex algebras in terms of generators and relations, thus giving an alternative construction of these algebras without using vertex operators. We remark that our construction works in a very general setting; we do not assume the lattice to be positive definite, neither non-degenerate, nor of a finite rank. 


\section{Organization of the manuscript}

We start with reviewing some basic definitions of vertex and conformal algebra in $\$ 1$ - $\$ 3$. The reader may consult Kac's book 11 for more details. Then in $\oint$ we recall the construction of the vertex algebra $V_{\Lambda}$ corresponding to an integer lattice $\Lambda$, the details can be again found in [11].

In $\$$ we construct the free vertex algebra $\mathfrak{F}_{N}(\mathcal{B})$ generated by a set $\mathcal{B}$ so that the order of locality $N(a, b)$ of a pair of generators $a, b \in \mathcal{B}$ is given by an arbitrary symmetric integer-valued function $N: \mathcal{B} \times \mathcal{B} \rightarrow \mathbb{Z}$. Theorem 1 describes a set $\mathcal{T} \subset \mathfrak{F}_{N}(\mathcal{B})$ and claims that $\mathcal{T}$ is a linear basis of $\mathfrak{F}_{N}(\mathcal{B})$. As a corollary, we find the dimensions of homogeneous components of $\mathfrak{F}_{N}(\mathcal{B})$. In $\S 11$ we will prove that $\mathcal{T}$ spans $\mathfrak{F}_{N}(\mathcal{B})$, and in $\$ 12$ we finish the proof of Theorem 11 by showing that $\mathcal{T}$ is linearly independent.

In $\$ 6$ we construct a vertex algebra homomorphism $\varphi: \mathfrak{F}_{N}(\mathcal{B}) \rightarrow V_{\Lambda}$ from the free vertex algebra $\mathfrak{F}_{N}(\mathcal{B})$ to the vertex algebra corresponding to the lattice $\Lambda=\mathbb{Z}[\mathcal{B}]$. The integer form on $\Lambda$ is defined by $(a \mid b)=-N(a, b)$ for $a, b \in \mathcal{B}$. Theorem 2 then states that $\varphi$ is injective. We will prove this theorem in $\S 12$.

In $\delta$, using Theorem 2, we prove a quantitative version of Dong's lemma. In $\$ 8$ we apply this lemma to settle a question raised in $\mid 16$ : we prove that the locality function of a free conformal algebra has quadratic growth.

In $\$ 9$ we study homogeneous conformal derivations of free vertex algebras. It turns out that a particularly interesting case is when the algebra is generated by a single element $a$ such that $N(a, a)=-1$. In this case $\mathfrak{F}_{N}(\{a\})$ is embedded into the fermionic vertex algebra $V_{\mathbb{Z}}$. We prove that a homogeneous component of $\varphi\left(\mathfrak{F}_{N}(\{a\})\right) \subset V_{\mathbb{Z}}$ is an irreducible lowest weight module over certain conformal algebra $\widehat{\mathfrak{W}} \subset V_{0}$, such that the coefficient algebra of $\widehat{\mathfrak{W}}$ is a central extension of the Lie algebra of differential operators on the circle, see [7, 11, 17.

Finally, in $\S 10$ we find a presentation of lattice vertex algebras in terms of generators and relations, see Theorem 4 . It turns out that the required relations are rather minimal. Our proof is completely combinatorial. The first step is to determine the structure of the vertex algebra in question as a module over the Heisenberg algebra.

\section{Notations}

All algebras and linear spaces are over a field $\mathbb{k}$ of characteristic 0 . Here are some shortcuts used throughout the paper: $\{n \in \mathbb{Z} \mid n \geqslant 0\}=\mathbb{Z}_{+}$, $\frac{1}{k !} n(n-1) \cdots(n-k+1)=\left(\begin{array}{l}n \\ k\end{array}\right), \quad \frac{1}{k !} D^{k}=D^{(k)}, \quad Z(L)$ is the center of a Lie algebra $L$. 


\section{Acknowledgements}

The core of this work was done while I was visiting the Fields Institute. I am very grateful to organizers of the Program on Lie Theory for inviting me there. I especially thank Stephan Berman, Yuly Billig and Chongying Dong for helpful discussions.

\section{FIELDS AND LOCALITY}

Let $V=V^{\overline{0}} \oplus V^{\overline{1}}$ be a vector superspace over $\mathbb{k}$. Take a formal variable $z$ and consider the space $\mathrm{F}(V)=\mathrm{F}(V)^{\overline{0}} \oplus \mathrm{F}(V)^{\overline{1}} \subset$ End $(V, V((z)))$ of fields on $V$, given by

$$
\mathrm{F}(V)^{p}=\left\{\sum_{n \in \mathbb{Z}} a(n) z^{-n-1} \mid p(a(n))=p, \forall v \in V, a(n) v=0 \text { for } n \gg 0\right\},
$$

Here $p(x) \in \mathbb{Z} / 2 \mathbb{Z}$ is the parity of $x$. Denote by $\mathbb{1} \in \mathrm{F}(V)^{\overline{0}}$ the identity operator, such that $\mathbb{1}(-1)=\operatorname{Id}_{V}$, all other coefficients are 0 .

Let $\imath_{w, z}(w-z)^{n}$ and $\imath_{z, w}(w-z)^{n}$ be two different expansions of $(w-z)^{n}$ into formal power series in the variables $w$ and $z$ :

$$
\begin{aligned}
& \imath_{w, z}(w-z)^{n}=\sum_{i \geqslant 0}(-1)^{n+i}\left(\begin{array}{c}
n \\
i
\end{array}\right) w^{n-i} z^{i} \in \mathbb{k}\left[\left[w, w^{-1}, z\right]\right], \\
& \imath_{z, w}(w-z)^{n}=\sum_{i \geqslant 0}(-1)^{i}\left(\begin{array}{c}
n \\
i
\end{array}\right) w^{i} z^{n-i} \in \mathbb{k}\left[\left[w, z, z^{-1}\right]\right] .
\end{aligned}
$$

Of course, if $n \geqslant 0$ then $\imath_{w, z}(w-z)^{n}=\imath_{z, w}(w-z)^{n}$.

Let $a, b \in \mathrm{F}(V)$. We say that $a$ is local to $b$ if there is some $N \in \mathbb{Z}$ such that

$$
a(w) b(z) \imath_{w, z}(w-z)^{N}-(-1)^{p(a) p(b)} b(z) a(w) \imath_{z, w}(w-z)^{N}=0 .
$$

The minimal $N=N(a, b)$ with this property is called the order of locality of $a$ and $b$. In terms of the coefficients locality means that the following identities hold for all $m, n \in \mathbb{Z}$ :

$$
\begin{aligned}
& \sum_{s \geqslant 0}(-1)^{s}\left(\begin{array}{c}
N \\
s
\end{array}\right) a(m-s) b(n+s) \\
& \quad-(-1)^{p(a) p(b)} \sum_{s \leqslant N}(-1)^{s}\left(\begin{array}{c}
N \\
N-s
\end{array}\right) b(n+s) a(m-s)=0
\end{aligned}
$$


Usually the locality is defined only for a nonnegative order $N$ 6, 11, but we will need this property in a bigger generality.

For an integer $n$ define a bilinear product $n: \mathrm{F}(V) \otimes \mathrm{F}(V) \rightarrow \mathrm{F}(V)$ by

$$
\begin{aligned}
& (a \square b)(z)=\operatorname{Res}_{w}\left(a(w) b(z) \imath_{w, z}(w-z)^{n}\right. \\
& \left.\quad-(-1)^{p(a) p(b)} b(z) a(w) \imath_{z, w}(w-z)^{n}\right) .
\end{aligned}
$$

Here $\operatorname{Res}_{w}$ stands for the coefficient of $w^{-1}$. Explicitly this means

$$
\begin{aligned}
(a \square b)(m) & =\sum_{s \geqslant 0}(-1)^{s}\left(\begin{array}{c}
n \\
s
\end{array}\right) a(n-s) b(m+s) \\
& -(-1)^{p(a) p(b)} \sum_{s \leqslant n}(-1)^{s}\left(\begin{array}{c}
n \\
n-s
\end{array}\right) b(m+s) a(n-s)
\end{aligned}
$$

Clearly, if $a$ and $b$ are local of order $N$ then $a \square b=0$ for $n \geqslant N$.

If $n=-1$, then $a-1 \quad b=: a b:=a_{-} b+b a_{+}$is the so-called normally ordered product, where

In general, if $n<0$ then

$$
a_{ \pm}(z)=\sum_{n \geqslant 0} a(n) z^{-n-1} .
$$

$$
\alpha \square \beta=:\left(D^{(-n-1)} \alpha\right) \beta:
$$

where $D \alpha(z)=\frac{d}{d z} \alpha(z)=\alpha(z)-2 \mathbb{1}$.

We remark that the following identities hold:

$$
(D a) \llbracket b=-n a n-1 b, \quad a \llbracket(D b)=n a n-1 b+D(a n b),
$$

in particular, $D$ is a derivation of the products $n$.

The Dong's lemma [6, 11, 13] states that if $a, b, c \in F(V)$ are three pairwise local fields, then for every $n \in \mathbb{Z}$ the fields $a n b$ and $c$ are local as well. In $\oint$ we will prove a quantitative version of this lemma.

A subspace $\mathfrak{A} \subset \mathrm{F}(V)$ such that

(i) any two fields $a, b \in \mathfrak{A}$ are local,

(ii) $\mathfrak{A} \square \mathfrak{A} \subseteq \mathfrak{A}$ for all $n \in \mathbb{Z}$ and

(iii) $\mathbb{1} \in \mathfrak{A}$ 
is called a vertex algebra. By the Dong's lemma, given a collection $\mathcal{S} \subset$ $\mathrm{F}(V)$ of pairwise local fields, the closure of $\mathcal{S} \cup\{\mathbb{1}\}$ under the products is a vertex algebra in $\mathrm{F}(V)$.

\section{AXIOMATIC DEFINITION OF VERTEX ALGEBRAS}

Vertex algebras can also be defined axiomatically as follows [11. Let $\mathfrak{A}=\mathfrak{A}^{\overline{0}} \oplus \mathfrak{A}^{\overline{1}}$ be a linear superspace endowed with a sequence of even bilinear operations $\square: \mathfrak{A} \otimes \mathfrak{A} \rightarrow \mathfrak{A}, n \in \mathbb{Z}$, and a distinguished element $\mathbb{1} \in \mathfrak{A}^{\overline{0}}$. Let $D: \mathfrak{A} \rightarrow \mathfrak{A}$ be an even linear map given by $D a=a-2 \mathbb{1}$. Consider the left regular action map $Y: \mathfrak{A} \rightarrow \mathfrak{A}\left[\left[z, z^{-1}\right]\right]$ defined by $Y(a)(z)=$ $\sum_{n \in \mathbb{Z}}(a n \cdot) z^{-n-1}$. Then $\mathfrak{A}$ is a vertex algebra if it satisfies the following conditions for any $a, b \in \mathfrak{A}$ and $n \in \mathbb{Z}$ :

V1. $a \square b=0$ for $n \gg 0$.

V2. $\mathbb{1} \square a=\delta_{n,-1} a, \quad a \square \mathbb{1}=\left\{\begin{array}{lll}0 & \text { if } & n \geqslant 0, \\ D^{(-n-1)} a & \text { if } & n<0 .\end{array}\right.$

V3. $(D a) \square b=-n a n-1 b, \quad a \square(D b)=n a n-1 b+D(a \square b)$.

V4. The series $Y(a), Y(b) \in \mathfrak{A}\left[\left[z, z^{-1}\right]\right]$ are local.

This is not a minimal set of axioms, in fact conditions V2 and V3 can be weakened. Note that V3 implies that $Y(D a)=\frac{d}{d z} Y(a)$. For other axiomatic definitions of vertex algebras see [2, 6, 8, 9].

A homomorphism of two vertex algebras $\mathfrak{A}$ and $\mathfrak{B}$ is a map $\phi: \mathfrak{A} \rightarrow \mathfrak{B}$ such that $\phi(\mathbb{1})=\mathbb{1}$ and $\phi(a \llbracket b)=\phi(a) \square \phi(b)$ for all $n \in \mathbb{Z}$, in particular $\phi(D a)=\frac{d}{d z} \phi(a)$. A module over a vertex algebra $\mathfrak{A}$ is a linear space $V$ such that there is a vertex algebra homomorphism $\phi: \mathfrak{A} \rightarrow \mathrm{F}(V)$ of $\mathfrak{A}$ into a vertex subalgebra of $\mathrm{F}(V)$. Similarly one defines the notions of ideals, quotients, isomorphisms, etc.

One of the most important properties of vertex algebras is that the left regular action map is in fact an isomorphism of a vertex algebra $\mathfrak{A}$ with a vertex subalgebra $Y(\mathfrak{A}) \subset \mathrm{F}(\mathfrak{A})$. In other words, the left regular action map is a faithful representation of $\mathfrak{A}$ on itself. In view of (4) this implies the following identity:

$$
\begin{aligned}
(a \square b) \amalg & =\sum_{s \geqslant 0}(-1)^{s}\left(\begin{array}{c}
n \\
s
\end{array}\right) a \underline{n-s}(b \underline{m+s} c) \\
& -(-1)^{p(a) p(b)} \sum_{s \leqslant n}(-1)^{s}\left(\begin{array}{c}
n \\
n-s
\end{array}\right) b \operatorname{m+s}(a[n-s c)
\end{aligned}
$$


for all $m, n \in \mathbb{Z}, a, b, c \in \mathfrak{A}$. An equivalent form of this identity is

$$
a \underline{m}(b \square c)-b \square(a \underline{m} c)=\sum_{s \geqslant 0}\left(\begin{array}{c}
m \\
s
\end{array}\right)(a \square b) m+n-s c .
$$

Another important idenity is the following so-called quasisymmetry identity:

$$
a \llbracket b=-\sum_{i \geqslant 0}(-1)^{n+i} D^{(i)}(b \longdiv { n + i } a) .
$$

There is a couple of additional axioms which are often imposed on vertex algebras, see [6, 8, 9, 13. A vertex algebra $\mathfrak{A}$ is called a vertex operator algebra or a conformal vertex algebra if

V5. $\mathfrak{A}=\bigoplus_{i \in \frac{1}{2} \mathbb{Z}} \mathfrak{A}_{i}$ is graded so that $\mathfrak{A}^{\overline{0}}=\sum_{i \in \mathbb{Z}} \mathfrak{A}_{i}, \quad \mathfrak{A}^{\overline{1}}=\sum_{i \in \mathbb{Z}+\frac{1}{2}} \mathfrak{A}_{i}$, $\mathbb{1} \in \mathfrak{A}_{0}$ and $\mathfrak{A}_{i} n \mathfrak{A}_{j} \subset \mathfrak{A}_{i+j-n-1}$.

V6. There exists an element $\omega \in \mathfrak{A}_{2}$ such that $N(\omega, \omega)=4, \omega 0 a=D a$, $\omega[a=(\operatorname{deg} a) a$ for all homogeneous $a \in \mathfrak{A}, \omega[\omega=0$ and $\omega \square \omega=c \mathbb{l}$ for some $c \in \mathbb{k}$.

The axiom V6 implies that $\omega$ generates the the Virasoro conformal algebra $\mathfrak{V} \mathfrak{i r} \subset \mathfrak{A}$ (see $\S 3$ below). The number $c$ is called the conformal charge of $\mathfrak{A}$.

We will call an expression of the form $a_{1} n_{1} \ldots n_{l-1} a_{l}, a_{i} \in \mathfrak{A}, n_{i} \in \mathbb{Z}$, (with an arbitrary order of parentheses) a vertex monomial of length $l$. Using (5) every vertex monomial can be expressed as a linear combination of right normed monomials of the form $a_{1} n_{1}\left(\cdots a_{l-2} n_{l-2}\left(a_{l-1} n_{l-1} a_{l}\right) \cdots\right)$. We will call a monomial $w$ minimal if every its submonomial $u n v$ has the maximal possible value of $n$, i.e. $n=N(u, v)-1$.

\section{CONFORMAL ALGEBRAS}

A conformal algebra is a more general algebraic structure than a vertex algebra, see 11]. The former is obtained from the latter by forgetting about $\mathbb{1}$ and the products $n$ for $n<0$. The identities V1, V3, (5) and (7) hold in a conformal algebra for $m, n \geqslant 0$.

Note that if $N \geqslant 0$ and $n \geqslant 0$, then (11) and (3) simplify respectively to

$$
\begin{gathered}
{[a(w), b(z)](w-z)^{N}=0,} \\
(a \square b)(z)=\operatorname{Res}_{w}[a(w), b(z)](w-z)^{n} .
\end{gathered}
$$

This means that the locality for $N \geqslant 0$ and the products $n$ for $n \geqslant 0$ both make sense for series $a, b \in L\left[\left[z^{ \pm 1}\right]\right]$ with coefficients in a Lie superalgebra 
$L$. Similar to the case of vertex algebras, a space $\mathfrak{L} \subset L\left[\left[z^{ \pm 1}\right]\right]$ of pairwise local series, such that $D \mathfrak{L} \subseteq \mathfrak{L}$ and $\mathfrak{L} \square \mathfrak{L} \subseteq \mathfrak{L}$ for $n \geqslant 0$, is a conformal algebra and all conformal algebras can be obtained in this way. The Dong's lemma holds for these series as well.

Moreover, for a given conformal algebra $\mathfrak{L}$ one can construct a Lie superalgebra $L=$ Coeff $\mathfrak{L}$, which is called the coefficient algebra of $\mathfrak{L}$, together with a conformal algebra homomorphism $\mathfrak{L} \rightarrow L\left[\left[z^{ \pm 1}\right]\right]$ which is universal among all representations of $\mathfrak{L}$ by formal series with coefficients in a Lie superalgebra. As a linear space $L$ is equal to $\mathfrak{L} \otimes \mathbb{k}\left[t^{ \pm 1}\right]$ modulo the linear subspace generated by all the expressions $(D a)(n)+n a(n-1)$ for $a \in \mathfrak{L}$ and $n \in \mathbb{Z}$. We denote here $a(n)=a \otimes t^{n}$.

\section{Examples}

Let $\mathfrak{g}$ be a Lie algebra with a symmetric invariant bilinear form $(\cdot \mid \cdot)$. Consider the corresponding affine Lie algebra $\tilde{\mathfrak{g}}=\mathfrak{g} \otimes \mathbb{k}\left[t^{ \pm 1}\right] \oplus \mathbb{k} \mathrm{c}$ with the brackets given by

$$
[a(m), b(n)]=[a, b](m+n)+\delta_{m,-n} m(a \mid b) c, \quad c \in Z(\mathfrak{g}) .
$$

This Lie algebra is the coefficient algebra of the conformal algebra $\mathfrak{G} \subset$ $\widetilde{\mathfrak{g}}\left[\left[z^{ \pm 1}\right]\right]$, generated by the series $\widetilde{a}=\sum_{n} a(n) z^{-n-1}$ for $a \in \mathfrak{g}$ and $\mathrm{c}=\mathrm{c}(-1)$ so that $D \mathrm{c}=0, N(\widetilde{a}, \widetilde{b})=1$ and

$$
\widetilde{a} \square \widetilde{b}=\widetilde{[a, b]}, \quad \widetilde{a} \square \widetilde{b}=(a \mid b) c .
$$

In the case when $\mathfrak{g}$ is an abelian Lie algebra, the corresponding affine algebra $\tilde{\mathfrak{g}}$ is a Heisenberg algebra, and $\mathfrak{G}$ is a Heisenberg conformal algebra.

Another example of a conformal algebra is obtained from the Virasoro Lie algebra Vir, which is spanned by the elements $L_{n}, n \in \mathbb{Z}$ and c with the brackets given by

$$
\left[L_{m}, L_{n}\right]=(m-n) L_{m+n}+\delta_{m,-n}\left(\begin{array}{c}
m+1 \\
3
\end{array}\right) \mathrm{c}, \quad \mathrm{c} \in Z(\text { Vir }) .
$$

It is the coefficient algebra of the conformal algebra $\mathfrak{V} \mathfrak{i r} \subset \operatorname{Vir}\left[\left[z^{ \pm 1}\right]\right]$ generated by the series $\omega=\sum_{n \in \mathbb{Z}} L_{n-1} z^{-n-1}$ and c. We have $D$ c $=0$, $N(\omega, \omega)=4$ and

$$
\omega \square \omega=D \omega, \quad \omega \square \omega=2 \omega, \quad \omega \square \omega=0, \quad \omega \square \omega=\mathrm{c} .
$$

Another example of a conformal algebra will be given in $\$ 9$. 


\section{Conformal operators}

Consider first a $\mathbb{k}[D]$-module $V$. A conformal operator [3] on $V$ is a series $\alpha=\sum_{n \in \mathbb{Z}_{+}} \alpha(n) z^{-n-1} \in z^{-1}(g l V)\left[\left[z^{-1}\right]\right]$ such that $\alpha(n) v=0$ if $n \gg 0$ for every fixed $v \in V$ and $[D, \alpha]=\frac{d}{d z} \alpha$. For $v \in V$ call $N(\alpha, v)=\min \left\{n \in \mathbb{Z}_{+} \mid \alpha(m) v=0 \forall m \geqslant n\right\}$ the order of locality of $\alpha$ and $v$. Denote by $\operatorname{cgl} V$ the space of all conformal operators on $V$.

A pair $\alpha, \beta \in c g l V$ is said to be local of order $N \in \mathbb{Z}_{+}$if (\&) holds. Also the products $\alpha \square \beta \in \operatorname{cgl} \mathfrak{A}$ are defined for $n \geqslant 0$ by the formula (9). The Dong's lemma also holds for conformal operators. A subspace $\mathfrak{L} \subset \operatorname{cgl} V$ of pairwise local conformal operators, closed under all the products $n$ for $n \in \mathbb{Z}_{+}$, is a conformal algebra. A module over a conformal algebra $\mathfrak{L}$ is a $\mathbb{k}[D]$-module $V$ with a conformal algebra homomorphism $\mathfrak{L} \rightarrow c g l V$.

It is well-known [3] that if $v \in V$ is such that $p(D) v=0$ for some polynomial $p \in \mathbb{k}[D]$ then $\alpha(n) v=0$ for every $\alpha \in \operatorname{cgl} V$ and $n \in \mathbb{Z}_{+}$. In particular, if $\mathfrak{A}$ is a vertex algebra, then $\alpha(n) \mathbb{1}=0$ for every $\alpha \in \operatorname{cgl} \mathfrak{A}$.

Let $\mathfrak{A}$ be a conformal (in particular, vertex) algebra. A conformal derivation [3, 16] is a conformal operator $\alpha \in \operatorname{cgl} \mathfrak{A}$ such that

$$
\alpha(m)(a n b)=a n(\alpha(m) b)+\sum_{s \geqslant 0}\left(\begin{array}{c}
m \\
s
\end{array}\right)(\alpha(s) a) m+n-s b
$$

for all $m \in \mathbb{Z}_{+}, n \in \mathbb{Z}$. For example, if $a \in \mathfrak{A}$, then

$$
Y(a)_{+}=\sum_{n \in \mathbb{Z}_{+}} Y(a)(n) z^{-n-1}
$$

is a conformal derivation of $\mathfrak{A}$. Denote by $\operatorname{cder} \mathfrak{A} \subset \operatorname{cgl} \mathfrak{A}$ the space of all conformal derivations of $\mathfrak{A}$. It is not difficult to show that if $\alpha, \beta \in \operatorname{cder} \mathfrak{A}$, then $\alpha n \beta \in \operatorname{cder} \mathfrak{A}$.

Assume that $\mathfrak{A}$ is generated by a set $\mathcal{B}$. Then a conformal derivation is uniquely defined by its action on $\mathcal{B}$. It is easy to show that if for $\alpha, \beta \in$ cder $\mathfrak{A}$ the orders of locality $N(\alpha, \mathcal{B}), N(\beta, \mathcal{B})$ are uniformly bounded on $\mathcal{B}$, then $\alpha$ and $\beta$ are local.

\section{LATTICE VERTEX ALGEBRAS}

In this section we give a very important example of vertex algebras the algebra $V_{\Lambda}$ corresponding to an integer lattice $\Lambda$. We mostly follow [1], see also [5, 6, 9]. Note, however, that we do not assume that $\Lambda$ is of a finite rank. 
Denote the integer-valued symmetric bilinear form on $\Lambda$ by $(\cdot \mid \cdot)$. Let us extend the form to $\mathfrak{h}=\Lambda \otimes_{\mathbb{Z}} \mathbb{k}$. Let $H=\widetilde{\mathfrak{h}}=\mathfrak{h} \otimes \mathbb{k}\left[t, t^{-1}\right] \oplus \mathbb{k} c$ be the corresponding Heisenberg algebra, see $\oint_{3}$.

For every $a \in \Lambda$ consider the canonical relation level 1 representation $V_{a}$ of $H$, that is, the irreducible Verma $H$-module generated by the vacuum vector $v_{a}$ such that $h(n) v_{a}=0$ for $n>0, h(0)=(h \mid a) \mathrm{Id}$, and $\mathrm{c}=\mathrm{Id}$, see [10]. It is well-known (see e.g. [11]) that the module $V_{0}$ has a structure of vertex algebra, such that $v_{0}=\mathbb{1}$. The map $\widetilde{h} \mapsto h(-1) v_{0}$ is an injective conformal algebra homomorphism of the conformal Heisenberg algebra $\mathfrak{H}$ to $V_{0}$. From now on we will identify $\mathfrak{H}$ with its image in $V_{0}$. In fact one can show that $V_{0}$ is a unique vertex algebra generated by $\mathfrak{H}$.

For $a \neq 0$, the map $\widetilde{h} \mapsto \sum_{n \in \mathbb{Z}} h(n) z^{-n-1} \in \mathrm{F}\left(V_{a}\right)$ is a conformal algebra homomorphism of $\mathfrak{H}$ into $\mathrm{F}\left(V_{a}\right)$, which extends to the vertex algebra homomorphism $V_{0} \rightarrow \mathrm{F}\left(V_{a}\right)$, so that $V_{a}$ becomes a module over the vertex algebra $V_{0}$. Define the Fock space

$$
V_{\Lambda}=\bigoplus_{a \in \Lambda} V_{a} \cong V_{0} \otimes \mathbb{k}[\Lambda]
$$

Let $\varepsilon: \Lambda \times \Lambda \rightarrow\{ \pm 1\}$ be a bimultiplicative map such that

$$
\varepsilon(a, b)=(-1)^{(a \mid a)(b \mid b)}(-1)^{(a \mid b)} \varepsilon(b, a) .
$$

for any $a, b \in \Lambda$. We remark that it is enough to check the identity (10) only when $a$ and $b$ belong to some $\mathbb{Z}$-basis of $\Lambda$; then (10) will follow for general $a, b$ by bimultiplicativity. The cocycle $\varepsilon$ defines an element of $H^{2}(\Lambda,\{ \pm 1\})$.

The main result 15, 6, 9, 11 is that the vertex algebra structure on $V_{0}$ can be uniquely extended to $V_{\Lambda}$ such that $\widetilde{h} \square v=h(n) v$ for any $h \in \mathfrak{h}$ and $v \in V_{\Lambda}$. The locality of a pair $v_{a}, v_{b} \in V$ of vacuum vectors is $N\left(v_{a}, v_{b}\right)=$ $-(a \mid b)$ and the products are

$$
v_{a}-(a \mid b)-k-1 \quad v_{b}=\varepsilon(a, b)(D-b(-1))^{(k)} v_{a+b}, \quad k \geqslant 0 .
$$

In particular,

$$
v_{a}-(a \mid b)-1 v_{b}=\varepsilon(a, b) v_{a+b}, \quad v_{a}-(a \mid b)-2 \quad v_{b}=\varepsilon(a, b) a(-1) v_{a+b} .
$$

Taking $b=-a$ we get

$$
v_{a}(a \mid a)-1 \quad v_{-a}=\varepsilon(a, a) \mathbb{1}, \quad v_{a}(a \mid a)-2 v_{-a}=\varepsilon(a, a) \tilde{a} .
$$

It follows that the vacuum vectors $v_{ \pm a}$, for $a$ running over an integer basis of $\Lambda$, generate the vertex algebra $V_{\Lambda}$, and (11) defines the vertex algebra 
structure on $V_{\Lambda}$ uniquely. The vertex algebra $V$ is simple if and only if the form $(\cdot \mid \cdot)$ is non-degenerate.

The standard way to construct vertex algebra $V_{\Lambda}$ is by showing that the vacuum vectors $v_{a}$ act under the left regular map $Y: V_{\Lambda} \rightarrow \mathrm{F}\left(V_{\Lambda}\right)$ by the so-called vertex operators. In $\$ 10$ we will construct $V_{\Lambda}$ by a different method.

Besides the grading by the lattice $\Lambda$, the vertex algebra $V_{\Lambda}$ has a grading by $\frac{1}{2} \mathbb{Z}$, so that $\operatorname{deg} v_{a}=\frac{1}{2}(a \mid a), \operatorname{deg} \widetilde{h}=1$ for every $h \in \mathfrak{h}, \operatorname{deg} \square=$ $-n-1$ and $\operatorname{deg} D=1$, in particular the axiom $V 5$ always holds. We have decomposition

$$
V_{a}=\bigoplus_{d \in \frac{1}{2}(a \mid a)+\mathbb{Z}_{+}} V_{a, d}, \quad V_{a, d} \square V_{a^{\prime}, d^{\prime}} \subset V_{a+a^{\prime}, d+d^{\prime}-n-1} .
$$

We will refer to the grading by $\Lambda$ as the grading by weights, and write wt $u=a \in \Lambda$ for $u \in V_{a}$.

Consider the case when $\Lambda$ is non-degenerate and of a finite rank $l$. Let $a_{1}, \ldots, a_{l}$ and $b_{1}, \ldots, b_{l}$ be dual bases of $\mathfrak{h}$, i.e. such that $\left(a_{i} \mid b_{j}\right)=\delta_{i j}$. Then the element $\omega=\frac{1}{2} \sum_{i=1}^{l} a_{i}-1 b_{i} \in V_{0}$ generates a copy of the Virasoro conformal algebra $\mathfrak{V i r}$ so that $\mathrm{V} 6$ is satisfied, and hence $V_{\Lambda}$ becomes a vertex operator algebra.

Remark. The correspondence $\Lambda \mapsto V_{\Lambda}$ is not a functor, because there is a certain degree of freedom in choosing the cocycle $\varepsilon$, satisfying (10). However, all the resulting vertex algebras $V_{\Lambda}$ are isomorphic.

\section{FREE VERTEX ALGEBRAS}

In this section we construct another example of vertex algebras - a free vertex algebra. Take some set of generators $\mathcal{B}$. We will assume that $\mathcal{B}$ is linearly ordered. Suppose we have a symmetric function $N: \mathcal{B} \times \mathcal{B} \rightarrow \mathbb{Z}$. Consider the category $\mathfrak{V e r}_{N}(\mathcal{B})$ of vertex algebras generated by the set $\mathcal{B}$ such that in any vertex algebra $\mathfrak{A} \in \mathfrak{V e r}_{N}(\mathcal{B})$ one has $a \square b=0$ for any $a, b \in \mathcal{B}$ whenever $n \geqslant N(a, b)$. We set the parity of $a \in \mathcal{B}$ to be $p(a) \equiv N(a, a) \bmod 2$. The morphisms of $\mathfrak{V e r}_{N}(\mathcal{B})$ are, naturally, vertex algebra homomorphisms $\phi: \mathfrak{A}_{1} \rightarrow \mathfrak{A}_{2}$ such that $\phi(a)=a$ for any $a \in \mathcal{B}$. Then it is easy to see that $\mathfrak{V e r}_{N}(\mathcal{B})$ has a unique universal object $\mathfrak{F}_{N}(\mathcal{B})$, called the free vertex algebra generated by $\mathcal{B}$ with respect to locality bound $N$. We construct $\mathfrak{F}_{N}(\mathcal{B})$ explicitly below.

Remark. It follows easily from the quazisymmetry identity (7) that if $a \in$ $\mathfrak{A}^{\overline{0}}$ is an even element of a vertex algebra $\mathfrak{A}$, then $N(a, a) \in 2 \mathbb{Z}$. 
Let $\mathfrak{A}$ be a vertex algebra generated by a set $\mathcal{B}$. For $a, b \in \mathcal{B}$ let

$$
N(a, b)=\min \{n \in \mathbb{Z} \mid a m b=0 \quad \forall m \geqslant n\}
$$

be the order of locality. Then there is a surjective homomorphism $\psi$ : $\mathfrak{F}_{N}(\mathcal{B}) \rightarrow \mathfrak{A}$. Let $\mathcal{R} \subset \mathfrak{F}_{N}(\mathcal{B})$ be a set of generators of Ker $\psi$. Then we say that $\mathfrak{A}$ is presented by generators $\mathcal{B}$, locality bound $N: \mathcal{B} \times \mathcal{B} \rightarrow \mathbb{Z}$ and relations $\mathcal{R}$.

We construct the free vertex algebra $\mathfrak{F}_{N}(\mathcal{B})$ as follows. Consider the set $\mathcal{X}=\{a(n) \mid a \in \mathcal{B}, n \in \mathbb{Z}\}$ and let $\mathbb{k}[\mathcal{X}]$ be the free associative algebra generated by $\mathcal{X}$. A module $M$ over $\mathbb{k}[\mathcal{X}]$ is called restricted if for any $a \in \mathcal{B}$ and $x \in M$ we have $a(n) x=0$ for $n \gg 0$. Let $\overline{\mathbb{k}[\mathcal{X}]}$ be the completion of $\mathbb{k}[\mathcal{X}]$ with respect to the topology in which any series $\sum_{i} p_{i}, \quad p_{i} \in \mathbb{k}[\mathcal{X}]$, that makes sense as an operator on every restricted module, converge. Let $I \subset \overline{\mathbb{k}[\mathcal{X}]}$ be the ideal generated by the locality relations (2) for all $a, b \in \mathcal{B}$, $N=N(a, b)$. Denote $U=\overline{\mathbb{k}[\mathcal{X}]} / I$. Let $\mathcal{X}_{+}=\{a(n) \mid a \in \mathcal{B}, n \geqslant 0\} \subset \mathcal{X}$ and consider the quotient $U / U \mathcal{X}_{+}$. This is a restricted left $U$-module, therefore any $a \in \mathcal{B}$ corresponds to a field $\sum_{n \in \mathbb{Z}} a(n) z^{-n-1} \in \mathrm{F}\left(U / U \mathcal{X}_{+}\right)$. These fields are local by the construction and hence they generate a vertex algebra $\mathfrak{F}_{N}(\mathcal{B}) \subset \mathrm{F}\left(U / U \mathcal{X}_{+}\right)$, which clearly satisfies all the criteria for being the desired free vertex algebra. By the Goddard-Kac existence theorem 11 , Theorem 4.5], the map $\phi \mapsto \phi(-1) 1$ is a one-to-one correspondence between $\mathfrak{F}_{N}(\mathcal{B})$ and $U / U \mathcal{X}_{+}$, so from now on we will identify the two.

In the special case when $N(a, b) \geqslant 0$ the locality identities (2) are finite sums of commutators, hence instead of $U$ one can consider an enveloping algebra of a certain Lie algebra. In this case the construction of $\mathfrak{F}_{N}(\mathcal{B})$ was done in [15]. It was also proved there that if $N \equiv N(a, b) \in 2 \mathbb{Z}_{+}$is a non-negative even constant, then a linear basis of $\mathfrak{F}_{N}(\mathcal{B})$ is given by all right normed vertex monomials

$$
a_{1} m_{1}\left(a_{2} m_{2} \cdots\left(a_{k}\left[m_{k} \mathbb{1}\right) \cdots\right), \quad a_{i} \in \mathcal{B}, m_{i} \in \mathbb{Z}, m_{k}<0,\right.
$$

such that

$$
m_{i}-m_{i+1} \leqslant \begin{cases}N & \text { if } a_{i} \leqslant a_{i+1} \\ N-1 & \text { otherwise }\end{cases}
$$

for $1 \leqslant i \leqslant k-1$. Note that if $m<0$, then $a \mathbb{m} \mathbb{1}=D^{(-m-1)} a$. In this paper we extend this result to the general case. 
Theorem 1. A linear basis of $\mathfrak{F}_{N}(\mathcal{B})$ is given by the set of all monomials (12) such that

$$
m_{i}-m_{i+1} \leqslant \begin{cases}\sum_{j=i+1}^{k} N\left(a_{i}, a_{j}\right)-\sum_{j=i+2}^{k} N\left(a_{i+1}, a_{j}\right) & \text { if } a_{i} \leqslant a_{i+1}, \\ \sum_{j=i+1}^{k} N\left(a_{i}, a_{j}\right)-\sum_{j=i+2}^{k} N\left(a_{i+1}, a_{j}\right)-1 & \text { otherwise }\end{cases}
$$

for $1 \leqslant i \leqslant k-1$.

Denote the set of all basic monomials by $\mathcal{T}$. We will prove this theorem in $\S 11$ and $\S 12$.

The free vertex algebra $\mathfrak{F}=\mathfrak{F}_{N}(\mathcal{B})$ is graded by the free abelian semigroup $\mathbb{Z}_{+}[\mathcal{B}]$, so that the weight of the monomial (12) is $a_{1}+\ldots+a_{k} \in$ $\mathbb{Z}_{+}[\mathcal{B}]$. Let $\mathfrak{F}_{\lambda}$ be the space of all elements of $\mathfrak{F}$ of weight $\lambda \in \mathbb{Z}_{+}[\mathcal{B}]$. We have the decomposition $\mathfrak{F}=\bigoplus_{\lambda \in \mathbb{Z}_{+}[\mathcal{B}]} \mathfrak{F}_{\lambda}$. There is also a grading by $\frac{1}{2} \mathbb{Z}$ such that $\operatorname{deg} b=-\frac{1}{2} N(b, b)$ for $b \in \mathcal{B}$. Each homogeneous subspace of weight $\lambda$ is in its turn decomposed $\mathfrak{F}_{\lambda}=\bigoplus_{d \in \frac{1}{2} \mathbb{Z}} \mathfrak{F}_{\lambda, d}$, so that

$$
\mathfrak{F}_{\lambda, d} \square \mathfrak{F}_{\lambda^{\prime}, d^{\prime}} \subset \mathfrak{F}_{\lambda+\lambda^{\prime}, d+d^{\prime}-n-1} \quad \text { and } \quad \mathbb{1} \in \mathfrak{F}_{0,0} .
$$

Note that the parity of a homogeneous element $w \in \mathfrak{F}$ is $p(w) \equiv 2 \operatorname{deg} w$ mod 2 so the axiom $\mathrm{V} 5$ holds. The basic set $\mathcal{T}$ is homogeneous with respect to both these gradings. Denote $\mathcal{T}_{\lambda, d}=\mathcal{T} \cap \mathfrak{F}_{\lambda, d}$.

Theorem 1 implies the following combinatorial meaning of the dimensions of homogeneous components of $\mathfrak{F}_{N}(\mathcal{B})$. Fix a weight $\lambda=\sum_{a \in \mathcal{B}} s_{a} a \in$ $\mathbb{Z}_{+}[\mathcal{B}]$. There is only one basic monomial $w_{\min }(\lambda) \in \mathcal{T}$ of weight $\lambda$ that attains the minimal possible degree $d_{\min }(\lambda)=\operatorname{deg} w_{\min }(\lambda)$. It is of the form

$$
w_{\min }(\lambda)=a_{1}\left[m_{1}\left(\cdots a_{k-2} m_{k-2}\left(a_{k-1} m_{k-1} a_{k}\right) \cdots\right),\right.
$$

where $a_{1} \leqslant a_{2} \leqslant \ldots \leqslant a_{k}$ and $m_{i}=\sum_{j=i+1}^{k} N\left(a_{i}, a_{j}\right)-1$ for $i \leqslant i \leqslant k-1$.

Let $w \in \mathcal{T}$ be a monomial, given by (12). Define a sequence of integers $\eta(w)=\left(n_{1}, \ldots, n_{k}\right) \in \mathbb{Z}^{k}$ where

$$
n_{i}=\sum_{j=i+1}^{k} N\left(a_{i}, a_{j}\right)-1-m_{j} \quad \text { for } 1 \leqslant i \leqslant k-1, \quad n_{k}=-1-m_{k} .
$$


By (13) we have $n_{1} \geqslant n_{2} \geqslant \ldots \geqslant n_{k}$ and if $n_{i}=n_{i+1}$ then $a_{i} \leqslant a_{i+1}$. Also, $\sum_{i} n_{i}=\operatorname{deg} w-\operatorname{deg}_{\min }($ wt $w)$. We can view $\eta(w)$ as a partition of the number $\operatorname{deg} w-\operatorname{deg}_{\min }($ wt $w)$ colored by the set $\mathcal{B}$ by setting the color of $n_{i}$ to be $a_{i}$. It is easy to see that this way we get a one-to-one correspondence between $\mathcal{T}_{\lambda, d}$ and the set of corresponding colored partitions of $d-d_{\min }(\lambda)$.

Corollary 1. The dimension of the homogeneous component $\mathfrak{F}_{N}(\mathcal{B})_{\lambda, d}$ is equal to the number of $\mathcal{B}$-colored partitions of $d-d_{\min }(\lambda)$ that contain at most $s_{a}$ terms of each color $a \in \mathcal{B}$.

In particular, these dimensions do not depend on the locality bound $N$, up to a shift.

\section{EMBEDDING OF FREE VERTEX ALGEBRAS INTO LATTICE VERTEX ALGEBRAS}

The two former sections indicate a striking similarity between lattice vertex algebras and free vertex algebras. In this section we make this similarity precise.

As in $\$$, let $\mathcal{B}$ be a set and let $N: \mathcal{B} \times \mathcal{B} \rightarrow \mathbb{Z}$ be a symmetric function. Let $\Lambda=\mathbb{Z}[\mathcal{B}]$ be the lattice generated by $\mathcal{B}$, define the bilinear form first for $a, b \in \mathcal{B}$ by $(a \mid b)=-N(a, b)$ and then extend it to the whole $\Lambda$ by linearity. By the universality property of the free vertex algebra $\mathfrak{F}=\mathfrak{F}_{N}(\mathcal{B})$ there is a vertex algebra homomorphism $\varphi: \mathfrak{F} \rightarrow V_{\Lambda}$ such that $\varphi(a)=v_{a}$ for each $a \in \mathcal{B}$. Note that $\varphi$ is homogeneous with respect to the double grading on $\mathfrak{F}$ and $V$. The following theorem claims that $\varphi$ is injective.

Theorem 2. Let $\mathcal{B} \subset \Lambda$ be a linearly independent set. Then the elements $v_{a}$ for $a \in \mathcal{B}$ generate a free vertex subalgebra in $V_{\Lambda}$.

We will prove this theorem in $\$ 12$. Here we deduce the following easy fact from the mere existence of the homomorphism $\varphi$ and from formula (111). Let $w=w_{\min }(\lambda) \in \mathfrak{F}$ be the basic minimal monomial of weight $\lambda=a_{1}+\ldots+a_{k}$ given by (14). Then it is easy to calculate that $\operatorname{deg} w=$ $\operatorname{deg}_{\min }($ wt $w)=\frac{1}{2}($ wt $w \mid$ wt $w)$.

Proposition 1. Let $w \in \mathfrak{F}_{\lambda}$ be monomial of weight $\lambda \in \mathbb{Z}_{+}[\mathcal{B}]$ such that any its submonomial $u$ has degree $\operatorname{deg} u=\operatorname{deg}_{\min }($ wt $u)=\frac{1}{2}$ (wt $u \mid$ wt $u$ ). Then $\varphi(w)= \pm v_{\lambda} \neq 0$.

Proof. Use induction on the length of $w$. If $w \in \mathcal{B}$, then $\varphi(w)=v_{\lambda}$ by the definition on $\varphi$. Otherwise, $w=u_{1} m u_{2}$ and by the induction, $\varphi\left(u_{i}\right)=$ $v_{\mathrm{wt} u_{i}}$. Since $\varphi$ is homogeneous, we have $\operatorname{deg} \varphi(w)=\operatorname{deg} w=\frac{1}{2}(\lambda \mid \lambda)$, hence $\varphi(w)=v_{\mathrm{wt}} u_{1}+\mathrm{wt} u_{2}=v_{\lambda}$. 
Note that we do not use either of Theorems 1 or 2 in the proof of Proposition 1 .

Clearly the minimal monomial $w_{\min }(\lambda)$ satisfies the assumption of this proposition. It will follow from Theorems 1 and 2 that every such monomial

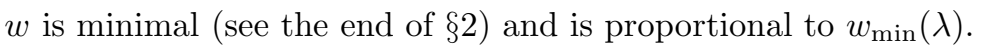

\section{QUANTITATIVE DONG'S LEMMA}

Let again $\mathcal{B}$ be a set with a locality function $N: \mathcal{B} \times \mathcal{B} \rightarrow \mathbb{Z}$ and let $\mathfrak{F}=\mathfrak{F}_{N}(\mathcal{B})$ be the corresponding free vertex algebra.

Lemma 1. Let $a, b, c \in \mathcal{B}$ and let $n=N(a, b)-k-1$ for some $k \geqslant 0$. Then

$$
N(c, b \square a)= \begin{cases}N(a, c)+N(b, c)+k & \text { if } \quad N(b, c)>0 \text { or } \\ & 0 \leqslant k \leqslant-N(b, c), \\ N(a, c) & \text { if } \quad N(b, c)=0 \text { or } \\ & 0<-N(b, c) \leqslant k .\end{cases}
$$

If $a, b, c$ belong to some arbitrary vertex algebra $\mathfrak{A}$ then clearly the locality $N(c, b \square a)$ is bounded from above by the right hand side of (16). Note that this estimate applies also to arbitrary fields $a, b, c \in \mathrm{F}(V)$, provided they are pairwise local. Indeed, by the qualitative version of Dong's lemma, such fields generate a vertex algebra in $\mathrm{F}(V)$.

Proof. By Theorem 2 it is enough to prove the lemma for the case when $a=v_{\alpha}, b=v_{\beta}$ and $c=v_{\gamma}$ are vacuum elements in a lattice vertex algebra $V_{\Lambda}$ for some vectors $\alpha, \beta, \gamma \in \Lambda$.

Let $m=-(\alpha+\beta \mid \gamma)+k-j-1$ for some $j \geqslant 0$. Using (11) together with the formulas

$$
\begin{gathered}
v_{\beta} \square(D u)=n v_{\beta} n-1 u+D\left(v_{\beta} \square u\right), \\
v_{\beta} \square(\alpha(-k) u)=-(\alpha \mid \beta) v_{\beta} n-k u+\alpha(-k)\left(v_{\beta} \square u\right),
\end{gathered}
$$

for $\alpha \in \mathfrak{h}, \beta \in \Lambda$ and $u \in V_{\Lambda}$, we obtain

$$
\begin{aligned}
c m(b \square a)= & \varepsilon(\alpha, \beta) \varepsilon(\alpha, \gamma) \varepsilon(\beta, \gamma) \sum_{i=k-j}^{k}\left(\begin{array}{c}
k-(\beta \mid \gamma)-j-1 \\
i
\end{array}\right) \\
& \times(D-\alpha(-1))^{(k-i)}(D-(\alpha+\beta)(-1))^{(j-k+i)} v_{\alpha+\beta+\gamma} .
\end{aligned}
$$


Let $j_{\text {min }}$ be the minimal value of $j$ such that $c m(b \square a) \neq 0$. We have

$$
N(c, b \square a)=-(\alpha+\beta \mid \gamma)+k-j_{\min }=N(a, c)+N(b, c)+k-j_{\min } .
$$

It follows that if $(\beta \mid \gamma)<0$ or if $(\beta \mid \gamma) \geqslant k \geqslant 0$, then $j_{\min }=0$; if $(\beta \mid \gamma)=0$, then $j_{\min }=k$; finally, if $k>(\beta \mid \gamma)>0$, then $j_{\min }=k-(\beta \mid \gamma)$ and the statement follows.

For monomials of length more than three the analogous estimate is more subtle. Note however, that by Corollary $\mathbb{1}$, if $w \in \mathfrak{F}$ is a vertex monomial in $\mathcal{B}$ such that $\operatorname{deg} w<\operatorname{deg}_{\text {min }}($ wt $w)$ then $w=0$. So we get:

Proposition 2. Let $w=a_{1} n_{1} \cdots \overline{n_{l-1}} a_{l} \in \mathfrak{F}, \quad a_{i} \in \mathcal{B}, n_{i} \in \mathbb{Z}$, be a vertex monomial. If

$$
\sum_{i=1}^{l-1} n_{i}>\sum_{1 \leqslant i<j \leqslant l} N\left(a_{i}, a_{j}\right)-l+1,
$$

then $w=0$.

Proposition 11 shows that sometimes the estimate $(17)$ is the best possible. For $l=3$ Lemma 1 gives in general a stronger estimate.

\section{LOCALITY FUNCTION}

Let $\mathfrak{L}$ be a conformal algebra (see $\delta[3$ ) generated by a finite set $\mathcal{B} \subset \mathfrak{L}$. In [16] the following integer function was defined. Consider all monomials $w=a_{1} n_{n} \cdots n_{l-1} a_{l} \in \mathfrak{L}, \quad a_{i} \in \mathcal{B}, n_{i} \in \mathbb{Z}_{+}$. Let $S(l)$ be the maximal possible value of $\sum_{i=1}^{l-1} n_{i}$ such that $w \neq 0$. We call $S$ the locality function of $\mathfrak{L}$. It depends on the generating set $\mathcal{B}$, however, if we take another generating set, then the growth of $S$ can change at most by a linear term. It was shown in $[16$ that if $\mathfrak{L}$ is embeddable into an associative conformal algebra, then $S(l)$ must have at most linear growth.

Clearly, similar function can be defined for a finitely generated vertex algebra.

Now let $\mathcal{B}$ be a finite set with a locality bound $N: \mathcal{B} \times \mathcal{B} \rightarrow \mathbb{Z}_{+}$that takes only non-negative values. Let $\mathfrak{F}=\mathfrak{F}_{N}(\mathcal{B})$ be the corresponding free vertex algebra (see $\$ \sqrt[5]{5}$ ). Let $\mathfrak{L} \subset \mathfrak{F}$ be the conformal algebra generated by $\mathcal{B}$. It follows from the results of 15 that $\mathfrak{L}$ is a free conformal algebra. It was shown in [16] that if $N \not \equiv 0$ then the locality function $S(l)$ of $\mathfrak{L}$ has at least quadratic growth, and hence $\mathfrak{L}$ cannot be embedded into an associative conformal algebra. It was also conjectured that $S(l)$ has exactly quadratic 
growth. Combining Propositions 1 and 2, we see that this conjecture is indeed true.

Corollary 2. The locality function of the free conformal algebra generated by a set $\mathcal{B}$ with locality bound $N$ is

$$
S(l)=\frac{l(l-1)}{2} \max _{a, b \in \mathcal{B}} N(a, b)-l+1 .
$$

\section{ACTIONS OF CONFORMAL ALGEBRAS ON FREE VERTEX ALGEBRAS}

Return to the setup of $\S$. Let $\mathfrak{F}=\mathfrak{F}_{N}(\mathcal{B})$ be the free vertex algebra generated by a set $\mathcal{B}$ with a locality bound $N: \mathcal{B} \times \mathcal{B} \rightarrow \mathbb{Z}$. Let $\Lambda=\mathbb{Z}[\mathcal{B}]$ and let $\varphi: \mathfrak{F} \rightarrow V_{\Lambda}$ be the injective homomorphism provided by Theorem 2 , such that $\varphi(a)=v_{a}$ for $a \in \mathcal{B}$.

We are interested in conformal derivations $\alpha \in \operatorname{cder} \mathfrak{F}$, which are homogeneous of weight 0 and of some degree $m+1 \in \mathbb{Z}$ (see $\S_{3}$ ). This means that for each $b \in \mathcal{B}$ we have $\alpha(n) b=f_{n}(b) D^{m-n} b$ for some functions $f_{n}: \mathcal{B} \rightarrow \mathbb{k}$ when $0 \leqslant n \leqslant m$, and $\alpha(n) b=0$ when $n>m$. Recall that a conformal derivation is uniquely defined by its action on the generators.

We study the case when $m=0$ in a greater detail, because it will be

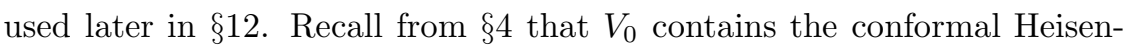
berg algebra $\mathfrak{H}$. This algebra acts on $V_{\Lambda}$ by commuting inner conformal derivations $\widetilde{h}_{+}$for $h \in \mathfrak{h}=\Lambda \otimes \mathbb{k}$ such that $N\left(\widetilde{h}_{+}, \widetilde{g}_{+}\right)=0$.

Lemma 2. (a) Let $f: \mathcal{B} \rightarrow \mathbb{k}$ be an arbitrary function. Then there is a unique conformal derivation $\alpha_{f} \in \operatorname{cder} \mathfrak{F}$ such that $N\left(\alpha_{f}, \mathcal{B}\right)=1$ and $\alpha_{f}(0) b=f(b) b$ for all $b \in \mathcal{B}$. For another function $g: N \rightarrow \mathbb{k}$ we have $N\left(\alpha_{f}, \alpha_{g}\right)=0$.

(b) Let $h \in \mathfrak{h}$ is such that $f(b)=(h \mid b)$ for all $b \in \mathcal{B}$. Then $\varphi\left(\alpha_{f}(n) x\right)=$ $h(n) \varphi(x)$ for every $x \in \mathfrak{F}$ and $n \geqslant 0$.

Proof. (a). Consider the derivation $\alpha_{f}(n), n \geqslant 0$, of the associative algebra $\overline{\mathbb{k}\langle\mathcal{X}\rangle}$ which acts on a generator $b(m) \in \mathcal{X}$ by $\alpha_{f}(n)(b(m))=f(b) b(m+n)$, see $\$$ for the notations. It is easy to see that $\alpha_{f}(n)$ preserves the ideal $I \subset \overline{\mathbb{k}\langle\mathcal{X}\rangle}$ generated by the locality relations (2), hence $\alpha_{f}(n)$ is a derivation of the algebra $U=\overline{\mathbb{k}\langle\mathcal{X}\rangle} / I$. Also, we have $\left[D, \alpha_{f}(n)\right]=-n \alpha_{f}(n-1)$ where $D$ is the derivation of $U$ defined by $D b(m)=-m b(m-1)$. Since $\alpha_{f}(n)$ can only increase the indices, it preserves the left ideal $U \mathcal{X}_{+} \subset U$, therefore $\alpha_{f}(n)$ acts on the free vertex algebra $\mathfrak{F}=U / U \mathcal{X}_{+}$. 
(b). Both $\alpha_{f}$ and $\widetilde{h}_{+}$are conformal derivations, therefore they are uniquely determined by their action on the generators $\mathcal{B}$. But for every $b \in \mathcal{B}$ we have $\alpha_{f}(n) b=\delta_{n, 0}(h \mid b) b$ and $h(n) v_{b}=\delta_{n, 0}(h \mid b) v_{b}$, and the claim follows.

Let us now consider the case when $m=1$. A derivation of weight 0 and degree 2 is determined by a pair of functions $f_{0}, f_{1}: \mathcal{B} \rightarrow \mathbb{k}$. One can show that in order for this derivation to be well defined $f_{0}$ must be constant. Take $f_{0} \equiv 1$ and an arbitrary function $f=f_{1}: \mathcal{B} \rightarrow \mathbb{k}$. This defines a conformal derivation $\omega_{f} \in \operatorname{cder} \mathfrak{F}$ such that $N\left(\omega_{f}, \mathcal{B}\right)=2$ and $\omega_{f}(0) b=D b, \quad \omega_{f}(1) b=f(b) b$ for every $b \in \mathcal{B}$. For another function $g: \mathcal{B} \rightarrow \mathbb{k}$ we have $N\left(\alpha_{f}, \omega_{g}\right)=N\left(\omega_{f}, \omega_{g}\right)=2$ and

$$
\omega_{f} \square \alpha_{g}=\frac{d}{d z} \alpha_{g}, \quad \omega_{f} \square \alpha_{g}=\alpha_{g}, \omega_{f} \square \omega_{g}=\frac{d}{d z} \omega_{g}, \quad \omega_{f} \square \omega_{g}=2 \omega_{g} .
$$

In particular each $\omega_{f}$ generates an action of the Virasoro conformal algebra $\mathfrak{V i r}$. Note that the products $\alpha_{f} \square \omega_{g}$ can be calculated using (1).

All these statements are proved in a way similar to the proof of Lemma 2 . Using Theorem 1 in $\$ 10$ one can show that the derivation $\omega_{f}$ can be extended from $\varphi(\mathfrak{F})$ to $V_{\Lambda}$ if and only if $f(b)=\frac{1}{2}(b \mid b)$. If $V_{\Lambda}$ has the Virasoro element $\omega \in V_{0}$, then this extension coincides with the inner conformal derivation $\omega_{+}$defined by $\omega$.

Remark. This shows that though free vertex algebras are not vertex operator algebras, they have an action of the conformal Virasoro algebra compatible with the $\frac{1}{2} \mathbb{Z}$-grading.

However, derivations of higher degree exist only in some very special cases.

\section{Bozon-fermion correspondence}

Suppose that $\mathcal{B}=\{a\}$ consists of only one element with $N(a, a)=-1$. Then $\Lambda=\mathbb{Z}$ and the form is given by $(m \mid n)=m n$. The corresponding lattice vertex algebra $V_{\mathbb{Z}}$ is the main object of the so-called bozon-fermion correspondence, see e.g. 111. It is well known that the Heisenberg vertex algebra $V_{0} \subset V_{\mathbb{Z}}$ contains a conformal algebra $\widehat{\mathfrak{W}}$ spanned over $\mathbb{k}[D]$ by the elements $p_{m}=v_{-1}{ }_{-m-1} v_{1}, m \in \mathbb{Z}_{+}$. The multiplication table is

$$
\begin{aligned}
& p_{m} \text { } p_{n}=\left(\begin{array}{c}
m+n-k \\
m
\end{array}\right) p_{m+n-k} \\
&-\sum_{s=0}^{m-k}(-1)^{k+s}\left(\begin{array}{c}
m+n-k-s \\
n
\end{array}\right) D^{(s)} p_{m+n-k-s}+\delta_{k, m+n+1}(-1)^{m} \mathbb{1} .
\end{aligned}
$$


In particular, $p_{0}=-\widetilde{a}$ generates the Heisenberg conformal algebra $\mathfrak{H} \subset \widehat{\mathfrak{W}}$, and $p_{1}=\frac{1}{2} \widetilde{a}-1 \widetilde{a}-\frac{1}{2} D \widetilde{a}$ generates the Virasoro conformal algebra $\mathfrak{V i r} \subset$ $\widehat{\mathfrak{W}}$.

The coefficient algebra of $\widehat{\mathfrak{W}}$ is a central extension $\widehat{W}=W \oplus \mathbb{k c}$ of the Lie algebra $W=\mathbb{k}\left\langle p, t^{ \pm 1} \mid[t, p]=1\right\rangle^{[-]}$of differential operators on the circle. The subalgebra $W_{+} \subset \widehat{W}$ spanned by the coefficients $p_{m}(n)$ for $n \geqslant 0$ can be identified with the Lie algebra $\mathbb{k}\langle p, t \mid[t, p]=1\rangle^{[-]}$of differential operators on the disk so that $p_{m}(n)=\frac{1}{m !} p^{m} t^{n}$, see [1] 17].

Using (11) and the fact that $\varepsilon=1$ we calculate that

$$
p_{m}(n) v_{1}= \begin{cases}(-1)^{m} D^{(m-n)} v_{1} & \text { if } m \geqslant n \\ 0 & \text { otherwise }\end{cases}
$$

Therefore, the inner conformal derivations $\left(p_{m}\right)_{+} \in c d e r V_{\mathbb{Z}}$ are homogeneous of weight 0 and degree $m+1$. We will now show that $\left(p_{m}\right)_{+}$preserves $\varphi(\mathfrak{F})$, so by Theorem 2 it acts on $\mathfrak{F}$.

Each vacuum vector $v_{k} \in V_{\mathbb{Z}}$ is a lowest weight vector for the Lie algebra $W_{+}$, meaning that for a homogeneous $g \in W_{+}$one has $g v_{k}=0$ if $\operatorname{deg} g>0$ and $g v_{k}=\lambda(g) v_{k}$ for some weight $\lambda \in\left(W_{+}\right)_{0}^{*}$ if $\operatorname{deg} g=0$. It is shown in 17] that $v_{k}$ generates an irreducible lowest weight $W_{+}$-module $U\left(W_{+}\right) v_{k} \subset V_{k}$ of the lowest weight $\lambda_{k}$ defined by $\lambda_{k}\left(p_{m}(m)\right)=(-1)^{m}\left(\begin{array}{c}m+k \\ k\end{array}\right)$. It is also proved in [17] that dimension of the homogeneous component of $U\left(W_{+}\right) v_{k}$ of degree $d+k^{2} / 2$ is equal to the number of partitions of $d$ into at most $k$ terms, i.e. is exactly $\operatorname{dim} \mathfrak{F}_{k, d+k^{2} / 2}$. This motivates the following theorem.

Theorem 3. $\varphi\left(\mathfrak{F}_{k}\right)=U\left(W_{+}\right) v_{k}$ for every $k \geqslant 1$.

Proof. Using the dimension argument it is enough to prove only that $U\left(W_{+}\right) v_{k} \subseteq \varphi\left(\mathfrak{F}_{k}\right)$, though in fact the proof of the other inclusion is no more difficult.

Since $v_{k} \in \varphi(\mathfrak{F})$, it is enough to show that $W_{+} \varphi(\mathfrak{F}) \subset \varphi(\mathfrak{F})$. The Lie algebra $W_{+}$is spanned by $p_{m}(n)$ for $m, n \geqslant 0$. From (18) it follows that $p_{m}(n) v_{1} \in \varphi(\mathfrak{F})$. Since $v_{1}$ is a generator of $\varphi(\mathfrak{F})$, it follows that each $p_{m}(n)$ preserves $\varphi(\mathfrak{F})$.

Remark. One can show that homogeneous conformal derivations of weight 0 and degree $m>2$ of a free vertex algebra $\mathfrak{F}$ exist if and only if $\mathfrak{F}$ is a tensor product of free vertex algebras, generated by a single generator $a$ such that either $N(a, a)=0$ or $N(a, a)=-1$. 


\section{PRESENTATION OF LATTICE VERTEX ALGEBRAS IN TERMS OF GENERATORS AND RELATIONS}

In this section we start with a lattice vertex algebra $V_{\Lambda}$ corresponding to an integer lattice $\Lambda$. Choose a $\mathbb{Z}$-basis $\Pi$ of $\Lambda$. We may assume that $\varepsilon(a, a)=1$ for every $a \in \Pi$. By Theorem 2 the vertex subalgebra of $V_{\Lambda}$ generated by $\left\{v_{a} \mid a \in \Pi\right\}$ is isomorphic to a free vertex algebra. On the other hand, it follows from (11) that if we complete these generators by $v_{-a}$ 's, then the resulting vertex algebra will be the whole $V_{\Lambda}$.

Theorem 4. The lattice vertex algebra $V_{\Lambda}$ is presented by generators $\left\{v_{a} \mid a \in \pm \Pi\right\}$ with locality bound given by $N\left(v_{a}, v_{b}\right)=-(a \mid b)$ and relations $\left\{v_{a}(a \mid a)-1 \quad v_{-a}=\mathbb{1} \mid a \in \Pi\right\}$.

Note that the quasisymmetry identity (7) implies that also $v_{-a}(a \mid a)-1 v_{a}=$ $\mathbb{1}$ for $a \in \Pi$.

For the proof we need the following lemma.

Lemma 3. The lattice vertex algebra $V_{\Lambda}$ is presented by generators $\widetilde{a}$ and $v_{ \pm a}$ for $a \in \Pi$ with locality bound given by

$$
N\left(v_{a}, v_{b}\right)=-(a \mid b), \quad N(\widetilde{a}, \widetilde{b})=2, \quad N\left(\widetilde{a}, v_{b}\right)=1,
$$

and the following set of relations:

(i) $\widetilde{a} \square \widetilde{b}=0, \widetilde{a} \square \widetilde{b}=(a \mid b) \mathbb{1}$,

(ii) $\widetilde{a} \square v_{b}=(a \mid b) v_{b}$,

(iii) $v_{a}(a \mid a)-1 \quad v_{-a}=\mathbb{1}$,

(iv) $D v_{a}=\widetilde{a}-1 v_{a}$,

for any $a, b \in \pm \Pi$.

The relations (i) mean that $\widetilde{a}$ 's generate the Heisenberg vertex algebra $\mathfrak{H} \subset V_{\Lambda}$. The relations (ii) say that each $v_{b}$ is a vacuum vector for the representation of the corresponding Heisenberg Lie algebra of weight given by $a \mapsto(a \mid b)$.

We first show how this lemma implies the theorem.

Proof of Theorem Q Consider a set of generators $\mathcal{B}=\left\{X_{a}, X_{-a} \mid a \in \Pi\right\}$ with the locality bound $N: \mathcal{B} \times \mathcal{B} \rightarrow \mathbb{Z}$ given by $N\left(X_{a}, X_{b}\right)=-(a \mid b)$. Let $\mathfrak{F}=\mathfrak{F}_{N}(\mathcal{B})$ be the corresponding free vertex algebra. For $a \in \pm \Pi$ denote $K_{a}=X_{a}(a \mid a)-1 X_{-a}, H_{a}=X_{a}(a \mid a)-2, X_{-a} \in \mathfrak{F}$. Let $\mathfrak{I} \subset \mathfrak{F}$ be the ideal generated by the relations $K_{a}=\mathbb{1}$ for $a \in \Pi$. We have to show that the 
quotient vertex algebra $\mathfrak{A}=\mathfrak{F} / \mathfrak{I}$ is isomorphic to $V_{\Lambda}$ so that $X_{a} \mapsto v_{a}$ under the projection map $\pi: \mathfrak{F} \rightarrow \mathfrak{F} / \mathfrak{I}=\mathfrak{A}$.

By Lemma 3 it is enough to show that the elements $\widetilde{a}=\pi\left(H_{a}\right), v_{a}=$ $\pi\left(X_{a}\right) \in \mathfrak{A}$ satisfy the identities (i)-(iv) above. Note that the locality of these elements is already as prescribed by (19), and (iii) holds by the assumption. The identities (i), (ii) and (iv) follow from the following identities that hold in the free vertex algebra $\mathfrak{F}$ for all $a, b \in \pm \Pi$ :

$$
\begin{aligned}
H_{a} \llbracket H_{b} & =(a \mid b) K_{a} k-2 K_{b}, \quad \text { for } k=0,1, \\
H_{a} \square X_{b} & =(a \mid b) K_{a}-1-X_{b}, \\
H_{a} \text {-1 } X_{a} & =X_{a}-2-K_{a}+(a \mid a) K_{a}-2-X_{a} .
\end{aligned}
$$

It is quite easy to prove these identities by straightforward calculations using only the construction of $\mathfrak{F}$ given in $\oint$ 国. However, we can simplify these calculations even further by using Theorem 2. Consider the set $\bar{\Pi}=$ $\{a, \bar{a} \mid a \in \Pi\}$ and extend the symmetric bilinear form to $\bar{\Lambda}=\mathbb{Z}[\bar{\Pi}]$ by $(a \mid \bar{b})=-(a \mid b),(\bar{a} \mid \bar{b})=(a \mid b)$. In the same way we extend the cocycle $\varepsilon$, assuming that $\varepsilon(a, \bar{a})=\varepsilon(\bar{a}, \bar{a})=1$ for $a \in \Pi$. Let $V_{\bar{\Lambda}}$ be the corresponding lattice vertex algebra and let $\varphi: \mathfrak{F} \rightarrow V_{\bar{\Lambda}}$ be the injective homomorphism constructed in $\S 6$, such that $\varphi\left(X_{a}\right)=v_{a}, \varphi\left(X_{-a}\right)=v_{\bar{a}}$ for $a \in \Pi$. Let us, for example, prove the first identity in (20), the other two are proved in the same way.

Take some $a, b \in \Pi$. By (11) we have $\varphi\left(K_{a}\right)=v_{a+\bar{a}}$ and $\varphi\left(H_{a}\right)=$ $a(-1) v_{a+\bar{a}}$, so, using (5) and (6), we get for any $k \in \mathbb{Z}_{+}$:

$$
\begin{aligned}
\varphi\left(H_{a}\right) \llbracket \varphi\left(H_{b}\right) & =\left(a(-1) v_{a+\bar{a}}\right) \llbracket\left(b(-1) v_{b+\bar{b}}\right) \\
& =\sum_{s \geqslant 0} a(-s-1) v_{a+\bar{a}}(k+s) b(-1) v_{b+\bar{b}} \\
& +\sum_{s \geqslant 0} v_{a+\bar{a}}(k-s-1) a(s) b(-1) v_{b+\bar{b}} .
\end{aligned}
$$

Since $(b \mid a+\bar{a})=(a \mid b+\bar{b})=0$ we have $\left[b(i), v_{a+\bar{a}}(j)\right]=a(0) v_{b+\bar{b}}=0$, so the only non-zero term in the above comes from the second sum when $s=1$ and we precisely get the first identity in (20).

\section{Proof of Lemma 目}

Let $\mathfrak{A}$ be the vertex algebra presented by the generators $\left\{\widetilde{a}, v_{ \pm a} \mid a \in \Pi\right\}$ with locality bound (19) and relations (i)-(iv). Since these relations hold in the lattice vertex algebra $V_{\Lambda}$, there is a projection $\pi: \mathfrak{A} \rightarrow V_{\Lambda}$. 
Let $w=v_{b_{1}} n_{1} \cdots n_{k-1} v_{b_{k}} \in \mathfrak{A}, \quad b_{i} \in \pm \Pi$, be a vertex monomial. Denote by $\Xi(w)$ the set of all vertex monomials involving the same indeterminates $v_{b_{i}}$ 's in the same order as in $w$ and with the same arrangement of parentheses. Let $\mu(w) \in \Xi(w)$ be the unique minimal monomial in $\Xi(w)$, see $\S 2$. As in Proposition 1, we have $\pi(\mu(w))= \pm v_{\mathrm{wt} w} \in V_{\Lambda}$.

Recall from $\S$ that $V_{\Lambda}$ is a module over the Heisenberg algebra $H=$ $\mathfrak{h} \otimes \mathbb{k}\left[t, t^{-1}\right] \oplus \mathbb{k} \mathfrak{c}$ for $\mathfrak{h}=\Lambda \otimes_{\mathbb{Z}} \mathbb{k}$. As before we denote $a \otimes t^{n}=a(n)$. The algebra $H=H_{-} \oplus H_{+}$is decomposed into a direct sum of subalgebras $H_{-}=\{h(n) \mid h \in \mathfrak{h}, n<0\} \oplus \mathbb{k} c$ and $H_{+}=\{h(n) \mid h \in \mathfrak{h}, n \geqslant 0\}$. Let $\widehat{H}_{-}=H_{-} \oplus \mathbb{k} D$ be the Lie algebra obtained from $H_{-}$by adjoining the derivation $D$ acting by $h(n) \mapsto-n h(n-1)$ and $D c=0$. Let $\widehat{H}=\widehat{H}_{-} \oplus H_{+}$. Then $V_{\Lambda}$ is a module over $\widehat{H}$.

For a linear combination $h=\sum_{a \in \Pi} s_{a} a \in \mathfrak{h}, s_{a} \in \mathbb{k}$, denote $\widetilde{h}=$ $\sum_{a \in \Pi} s_{a} \widetilde{a} \in \mathfrak{A}$. We observe that $\mathfrak{A}$ is also a module over $\widehat{H}$ with the action given by $h(n) x=\widetilde{h} \square x$, and the vertex algebra homomorphism $\pi: \mathfrak{A} \rightarrow V_{\Lambda}$ is also a $\widehat{H}$-module homomorphism.

Lemma 4. Let $w=\mu(w) \in \mathfrak{A}$ be a right-normed minimal monomial of weight $\lambda \in \Lambda$. Then

(a) For any $a \in \pm \Pi$, we have $N\left(v_{a}, w\right)=-(a \mid \lambda)$.

(b) $D w=\lambda(-1) w$.

Proof. We prove this lemma by induction on the length $l$ of $w$. If $l=1$ then $w=v_{\lambda}$ and statements (a) and (b) are true by assumption.

Assume $l>1$. Then $w=v_{b} n u$ for some other maximal right-normed monomial $u$ of weight $\lambda^{\prime}=\lambda-b$. By the induction hypothesis, $N\left(v_{b}, u\right)=$ $-\left(b, \lambda^{\prime}\right)=n+1$. Using Lemma 1, we have: $N\left(v_{a}, v_{b} \square u\right)=-(a \mid b)-$ $\left(a \mid \lambda^{\prime}\right)-\left(b \mid \lambda^{\prime}\right)-n-1=-(a \mid \lambda)$. This proves (a).

In order to prove (b) we first calculate, using (5):

$$
\begin{aligned}
\left(b(-1) v_{b}\right) \square u & =\sum_{s<0} b(s)\left(v_{b} n-s-1 u\right)+\sum_{s \geqslant 0} v_{b} n-s-1(b(s) u) \\
& =b(-1)\left(v_{b} n u\right)+\left(b \mid \lambda^{\prime}\right) v_{b} n-1 u .
\end{aligned}
$$

Now we have, using the induction hypethesis and (6),

$$
\begin{aligned}
D\left(v_{b} \square u\right)= & \left(b(-1) v_{b}\right) \square u+v_{b} \square\left(\lambda^{\prime}(-1) u\right) \\
= & b(-1)\left(v_{b} n u\right)+\left(b \mid \lambda^{\prime}\right) v_{b} n-1 u \\
& \quad-\left(b \mid \lambda^{\prime}\right) v_{b} n u+\lambda^{\prime}(-1)\left(v_{b} \square u\right) \\
= & \lambda(-1)\left(v_{b} n u\right) .
\end{aligned}
$$


An element $x \in \mathfrak{A}$ is called vacuum, if for every $h \in \mathfrak{h}$ one has $h(n) x=0$ if $n>0$, and $h(0) x=\lambda(h) x$ for some $\lambda \in \mathfrak{h}^{*}$. It is easy to see that a minimal monomial $w=\mu(w) \in \mathfrak{A}$ is a vacuum element. We prove next that the minimal monomials generate $\mathfrak{A}$ over the extended Heisenberg algebra $\widehat{H}$. It will follow then by the representation theory of Heisenberg algebras (see e.g. [10]) that as a module over $\widehat{H}$ the vertex algebra $\mathfrak{A}=$ $\bigoplus_{w=\mu(w)} U(\widehat{H}) w$ is decomposed into a direct sum of irreducible highest weight modules generated by minimal monomials. Recall that by (5) a vertex algebra is spanned by right-normed monomials in its generators.

Lemma 5. Let $w \in \mathfrak{A}$ be a right-normed monomial in the variables $\left\{v_{a} \mid a \in \pm \Pi\right\}$. Then $w \in U\left(\widehat{H}_{-}\right) \mu(w)$.

Proof. As before we prove this lemma by induction on the length $l$ of $w$. If $l=1$, then $w=\mu(w)=v_{a}$. Otherwise, $w=v_{a} \square u$ for some right-normed monomial $u$. By induction, we have $u=g \mu(u)$ for some $g \in U\left(\widehat{H}_{-}\right)$. Applying the formulas

$$
\begin{gathered}
v_{a} \square(D u)=n v_{a} n-1 u+D\left(v_{a} \square u\right), \\
v_{a} \square(h(-k) u)=-(a \mid h) v_{a} n-k u+h(-k)\left(v_{a} \square u\right), \quad h \in \mathfrak{h},
\end{gathered}
$$

which follow from $\mathrm{V} 3$ and (6), we can express $w$ as

$$
w=\sum_{j \in \mathbb{Z}} g_{j} v_{a}\left\lceil\mu(u), \quad g_{j} \in U\left(\widehat{H}_{-}\right) .\right.
$$

Thus we have reduced the lemma to the case when $w=v_{a} n u$ for a minimal monomial $u=\mu(u)$.

Let $\lambda=$ wt $u \in \Lambda$. By Lemma A(a), we have $N\left(v_{a}, u\right)=-(a \mid \lambda)$. Assume that $w$ is not maximal, then $n=-(a \mid \lambda)-k-1$ for some $k>0$. Using Lemma $1(\mathrm{~b})$ and the formulas above, we get

$$
\begin{aligned}
& \lambda(-1)\left(v_{a} n+1 u\right)=(a \mid \lambda) v_{a} n u+v_{a} n+1 D u \\
& =(a \mid \lambda) v_{a} n u+(n+1) v_{a} \square u+D\left(v_{a} n+1 u\right) \\
& =D\left(v_{a} n+1 u\right)-k v_{a} \square u \text {. }
\end{aligned}
$$

Therefore, $\left.v_{a} \square u=\frac{1}{k}(D-\lambda(-1))\left(v_{a} n+1\right]\right)$, and the lemma follows.

Note that here we already recover the formula (11), up to a sign. 
As in the proof of Theorem 4 , consider the free vertex algebra $\mathfrak{F}=\mathfrak{F}_{N}(\mathcal{B})$ generated by the set $\mathcal{B}=\left\{X_{a} \mid a \in \pm \Pi\right\}$. Recall that $\mathfrak{F}$ is graded by the semilattice $\bar{\Lambda}_{+}=\mathbb{Z}_{+}[\bar{\Pi}]$, where $\bar{\Pi}=\{a, \bar{a} \mid a \in \Pi\}$, so that wt $X_{a}=a$, wt $X_{-a}=\bar{a}$ for $a \in \Pi$. Let $\psi: \mathfrak{F} \rightarrow \mathfrak{A}$ be the vertex algebra homomorphism such that $\psi\left(X_{a}\right)=v_{a}$ for $a \in \pm \Pi$.

By Corollary 1, all monomials in $\mathfrak{F}$ of a weight $\lambda \in \bar{\Lambda}_{+}$having the minimal possible degree $d_{\min }(\lambda)$ are proportional to $w_{\min }(\lambda)$, given by (14). If $\lambda$ contains a pair $a, \bar{a}$, then by permuting the variables in $w_{\min }(\lambda)$ we obtain a minimal monomial of the form $u \square\left(v_{a}(a \mid a)-1 v_{-a}\right) \in \mathfrak{F}_{\lambda}$. Applying (iii) we get that $\psi\left(w_{\min }(\lambda)\right)$ is proportional to $\psi\left(w_{\min }(\lambda-a-\bar{a})\right)$ in $\mathfrak{A}$. It follows that minimal monomials in $\mathfrak{A}$ in the variables $\left\{v_{a} \mid a \in \pm \Pi\right\}$ are parametrized by the lattice $\Lambda$. Combining this with Lemma 5 , we see that the vertex algebra $\mathfrak{A}$ is decomposed into a direct sum $\mathfrak{A}=\bigoplus_{\lambda \in \Lambda} \mathfrak{A}_{\lambda}$ of irreducible highest weight $\widehat{H}$-modules $\mathfrak{A}_{\lambda}$. Since $V_{\Lambda}$ has the same decomposition, the projection $\pi: \mathfrak{A} \rightarrow V_{\Lambda}$ must be an isomorphism. This finishes the proof of Lemma 3 .

Remark. In fact it is not very difficult to make the last argument without a reference to Corollary 1, thus rendering the whole proof of Theorem 10 more or less independent on Theorems 1 and 2 .

Remark. One can prove Lemma 3 in a different way, using the construction of $V_{\Lambda}$ by vertex operators, and the so-called method of $Z$-algebras, that originates to the work of Lepowsky and Wilson [12], see also [5, 9, 14]. This remark is due to C. Dong.

\section{PROOF OF THEOREM 1, PART I}

Let as before $\mathcal{B}$ be a set with a symmetric locality bound $N: \mathcal{B} \times \mathcal{B} \rightarrow \mathbb{Z}$ and let $\mathfrak{F}=\mathfrak{F}_{N}(\mathcal{B})$ be the corresponding free vertex algebra. Let $\mathcal{X}=$ $\{a(n) \mid a \in \mathcal{B}, n \in \mathbb{Z}\}$. As in 9 罒, consider the completion $\overline{\mathbb{k}\langle\mathcal{X}\rangle}$ of the free associative algebra generated by $\mathcal{X}$. Every element $g \in \overline{\mathbb{k}\langle\mathcal{X}\rangle}$ is a linear combination, possibly infinite, of words $\mathcal{X}^{*}$ in the alphabet $\mathcal{X}$.

By $\left\{\right.$ 姃 there is a map $\rho: \overline{\mathbb{k}\langle\mathcal{X}\rangle} \rightarrow \mathfrak{F}$, defined by $a_{1}\left(n_{1}\right) \cdots a_{k}\left(n_{k}\right) \mapsto$ $a_{1} n_{1}\left(a_{2} n_{2} \cdots\left(a_{k} n_{n} \mathbb{1}\right) \cdots\right)$. Under this map the set $\mathcal{T}$, described by Theorem 1, can be identified with the set of words $a_{1}\left(n_{1}\right) \cdots a_{k}\left(n_{k}\right) \in \mathcal{X}^{*}$ satisfying the condition (13). In this section we prove that $\rho \mathcal{T}$ spans $\mathfrak{F}$ over $\mathbb{k}$.

Let $w \in \mathcal{X}^{*}$ and $f \in \overline{\mathbb{k}\langle\mathcal{X}\rangle}$. A rule $w \stackrel{r}{\longrightarrow} f$ on $\overline{\mathbb{k}\langle\mathcal{X}\rangle}$ is a partially defined linear map $\overline{\mathbb{k}\langle\mathcal{X}\rangle} \rightarrow \overline{\mathbb{k}\langle\mathcal{X}\rangle}$ which is applicable to an element $g \in \overline{\mathbb{k}\langle\mathcal{X}\rangle}$ if $w$ occures in the decomposition of $g$ into a linear combination of words from 
$\mathcal{X}^{*}$. The result $h$ of the application of $r$ to $g$ is obtained by substituting $f$ instead of $w$; in this case we write $g \stackrel{r}{\longrightarrow} h$. For a set of rules $\mathcal{R}$ denote by $M(\mathcal{R})=\left\{w \in \mathcal{X}^{*} \mid \exists w \longrightarrow f \in \mathcal{R}\right\}$ the set of words to which at least one rule from $\mathcal{R}$ is applicable.

Now we construct a set of rules $\mathcal{R}$ that will correspond to the locality relations (2) in the generators $\mathcal{B}$. Let $w=a_{1}\left(n_{1}\right) \cdots a_{k}\left(n_{k}\right) \in \mathcal{X}^{*}$. Define

$$
\begin{aligned}
m_{j} & =\sum_{i=j+1}^{k} N\left(a_{j}, a_{i}\right)-\sum_{i=j+2}^{k} N\left(a_{j+1}, a_{i}\right) \quad \text { for } 1 \leqslant j \leqslant k-2, \\
m_{k-1} & =N\left(a_{k-1}, a_{k}\right) .
\end{aligned}
$$

Suppose there is $1 \leqslant j \leqslant k-1$ such that $n_{j}-n_{j+1}>m_{j}$ or $n_{j}-n_{j+1}=m_{j}$ and $a_{j}>a_{j+1}$. Then $\mathcal{R}$ contains the rule

$$
w \longrightarrow a_{1}\left(n_{1}\right) \cdots a_{j-1}\left(n_{j-1}\right) f_{j} a_{j+2}\left(n_{j+2}\right) \cdots a_{k}\left(n_{k}\right)
$$

for

$$
\begin{aligned}
f_{j}= & -\sum_{s \geqslant 1}(-1)^{s}\left(\begin{array}{c}
N \\
s
\end{array}\right) a_{j}\left(n_{j}-s\right) a_{j+1}\left(n_{j+1}+s\right) \\
& +(-1)^{p\left(a_{j}\right) p\left(a_{j+1}\right)} \sum_{s \leqslant N}(-1)^{s}\left(\begin{array}{c}
N \\
N-s
\end{array}\right) a_{j+1}\left(n_{j+1}+s\right) a_{j}\left(n_{j}-s\right),
\end{aligned}
$$

where $N=N\left(a_{j}, a_{j+1}\right)$.

We need another set $\mathcal{Q}$ of rules on $\overline{\mathbb{k}\langle\mathcal{X}\rangle}$. Consider a sequence of elements $a_{1}, \ldots, a_{k} \in \mathfrak{F}$. By the qualitative Dong's lemma there is $S=$ $S\left(a_{1}, \ldots, a_{k}\right) \in \mathbb{Z}$ such that any vertex monomial $w=a_{1} n_{1} \cdots n_{k-1} a_{k} \in$ $\mathfrak{F}_{N}(\mathcal{B})$ is equal to 0 whenever $\sum_{i=1}^{k-1} n_{i} \geqslant S$.

Remark. It will follow that $S\left(a_{1}, \ldots, a_{k}\right)=\sum_{1 \leqslant i<j \leqslant k} N\left(a_{i}, a_{j}\right)-k+2$, see Proposition 2. We could prove this right now, but our argument does not make use of this sharp estimate.

Define $\mathcal{Q}$ to be the set of all rules $w \longrightarrow 0$ such that $w=a_{1}\left(n_{1}\right) \cdots a_{k}\left(n_{k}\right)$ has the following property: there is $1 \leqslant j \leqslant k$ such that $\sum_{i=j}^{k} n_{i} \geqslant$ $S\left(a_{j}, \ldots, a_{k}, \mathbb{1}\right)$. In particular, if $n_{k} \geqslant 0$, then $w \longrightarrow 0 \in \mathcal{Q}$. We observe that the set $\mathcal{T}$ is exactly the set of all terminal monomials with respect to the rules $\widehat{\mathcal{R}}=\mathcal{R} \cup \mathcal{Q}$, i.e. the set of monomials to which these rules can not be applied. On the other hand, for any rule $w \longrightarrow f \in \widehat{\mathcal{R}}$ the identity $\rho(w-f)=0$ holds in $\mathfrak{F}$. Therefore, all we have to prove is that every word $w \in \mathcal{X}^{*}$ can be reduced to a linear combination of terminal 
words by a (possibly, infinite) number of applications of the rules $\widehat{\mathcal{R}}$. Thi sterminal linear combination is necessary finite, because the rules $\widehat{\mathcal{R}}$ preserve weight and degree, and there are only finitely many monomials in $\mathcal{T}$ of given weight and degree, see Corollary 1 .

We will apply the rules $\widehat{\mathcal{R}}$ to a word $w \in \mathcal{X}^{*}$ is such a way that $\mathcal{Q}$ has priority over $\mathcal{R}$, in other words, we apply $\mathcal{R}$ only if $\mathcal{Q}$ can no longer be applied. Denote by $\Omega(w)$ the set of all words $u \notin M(\mathcal{Q})$ that can appear in the process of applying the rules $\widehat{\mathcal{R}}$ to $w$. To prove that $\mathcal{T}$ spans $\mathfrak{F}$ it is enough to show the following two things:

(i) $w \notin \Omega(w)$;

(ii) $|\Omega(w)|<\infty$.

Indeed, if both (i) and (ii) are true, then the rules $\mathcal{R}$ can be applied to $w$ at most finitely many times, after that $w$ is reduced to a linear combination of $\mathcal{T}$.

Recall that the set of generators $\mathcal{B}$ is linearly ordered. Extend this order alphabetically to $\mathcal{B}^{*}$ by comparing the words from left to right. For a word $w=a_{1}\left(n_{1}\right) \cdots a_{k}\left(n_{k}\right) \in \mathcal{X}^{*}$ denote by $w_{i}=a_{i}\left(n_{i}\right) \cdots a_{k}\left(n_{k}\right)$ the $i$-th tail of $w$. Let also

$$
d(w)=-\sum_{i=1}^{k} n_{i}+\sum_{1 \leqslant i<j \leqslant k} N\left(a_{i}, a_{j}\right) .
$$

Take a word $u=b_{1}\left(p_{1}\right) \cdots b_{k}\left(p_{k}\right) \in \Omega(w)$. It is easy to see that $\left(b_{1}, \ldots, b_{k}\right)$ is a permutation of $\left(a_{1}, \ldots, a_{k}\right)$. Consider two sequences of integers: $\left(d\left(w_{1}\right), \ldots, d\left(w_{k}\right)\right)$ and $\left(d\left(u_{1}\right), \ldots, d\left(u_{k}\right)\right)$. We note that if $d(w) \ll 0$, then $w \in M(\mathcal{Q})$, therefore both claims (i) and (ii) follow from the following statement:

(iii) For every $1 \leqslant i \leqslant k$ we have $d\left(u_{i}\right) \leqslant d\left(w_{i}\right)$; moreover, if $\left(d\left(w_{1}\right), \ldots, d\left(w_{k}\right)\right)=\left(d\left(u_{1}\right), \ldots, d\left(u_{k}\right)\right)$, then $b_{1} \cdots b_{k}<a_{1} \cdots a_{k} \in \mathcal{B}^{*}$.

It is enough to check (iii) for a word $u$ that appears in the right-hand side of a rule $w \longrightarrow f \in \mathcal{R}$. Then $u$ is either

$$
a_{1}\left(n_{1}\right) \cdots a_{j-1}\left(n_{j-1}\right) a_{j}\left(n_{j}-s\right) a_{j+1}\left(n_{j+1}+s\right) a_{j+2}\left(n_{j+2}\right) \cdots a_{k}\left(n_{k}\right)
$$

for $s \geqslant 1$ or

$$
a_{1}\left(n_{1}\right) \cdots a_{j-1}\left(n_{j-1}\right) a_{j+1}\left(n_{j+1}+s\right) a_{j}\left(n_{j}-s\right) a_{j+2}\left(n_{j+2}\right) \cdots a_{k}\left(n_{k}\right)
$$

for $s \leqslant N=N\left(a_{j}, a_{j+1}\right)$. In both cases $d\left(u_{i}\right)=d\left(w_{i}\right)$ when $i \neq j+1$. In the first case we always have $d\left(u_{j+1}\right)=d\left(w_{j+1}\right)-s<d\left(w_{j+1}\right)$. In the 
second case

$$
\begin{aligned}
d\left(u_{j+1}\right) & =d\left(w_{j+1}\right)-\sum_{i=j+2}^{k} N\left(a_{j+1}, a_{i}\right)+\sum_{i=j+2}^{k} N\left(a_{j}, a_{i}\right)+n_{j+1}-n_{j}+s \\
& \leqslant d\left(w_{j+1}\right)+m_{j}-n_{j}+n_{j+1}
\end{aligned}
$$

where $m_{j}$ is given by (21). Now since $w \in M(\mathcal{R})$, we have $n_{j}-n_{j+1} \leqslant m_{j}$ and the equality can hold only if $a_{j}>a_{j-1}$. This proves (iii).

Remark. In fact we have shown that $\widehat{\mathcal{R}}$ is a rewriting system, which is a generalization of the rewriting system constructed in 15 for the case when the locality bound is constant and non-negative. Only minor modifications to the argument in 15] are needed to show that $\widehat{\mathcal{R}}$ is confluent, i.e. the final result of applications of the rules does not depend on the order in which the rules are applied. Then the Diamond lemma [1] 15] would imply that $\rho \mathcal{T}$ is a basis of $\mathfrak{F}$. However, in $\$ 12$ we prove that $\rho \mathcal{T}$ is linearly independent in $\mathfrak{F}$ by a different method.

\section{PROOF OF THEOREM 1, PART II AND PROOF OF THEOREM 2}

Let $\mathfrak{F}=\mathfrak{F}_{N}(\mathcal{B})$ be a free vertex algebra. Recall that there is a homomorphism $\varphi: \mathfrak{F} \rightarrow V_{\Lambda}$ for $\Lambda=\mathbb{Z}[\mathcal{B}]$, see $\S 6$. Denote also $\Lambda_{+}=$ $\mathbb{Z}_{+}[\mathcal{B}]$. In $\delta 11$ we have proved that the image of the set $\mathcal{T} \in \mathcal{X}^{*}$ of words $a_{1}\left(n_{1}\right) \cdots a_{k}\left(n_{k}\right) \in \mathcal{X}^{*}$ satisfying the condition (13) spans $\mathfrak{F}$ over $\mathbb{k}$ under the projection $\rho: \overline{\mathbb{k}\langle\mathcal{X}\rangle} \rightarrow \mathfrak{F}$. As before we will identify $\mathcal{T}$ with $\rho \mathcal{T}$. In this section we prove that $\varphi \mathcal{T} \subset V_{\Lambda}$ is linearly independent over $\mathbb{k}$. Combined with $\S 11$ this will prove both Theorem 11 and Theorem 2 .

Step 1. First of all we note that without a loss of generality we can assume that the form $(\cdot \mid \cdot)$ is non-degenerate on $\Lambda$. For otherwise we can embed the set $\mathcal{B}$ into a bigger set $\overline{\mathcal{B}}$ preserving the locality bound $N$, such that the matrix $\{N(a, b)\}_{a, b \in \overline{\mathcal{B}}}$ is non-degenerate. Then, if we have proved the statement for the non-degenerate case, we know that the set $\mathcal{T}$ in linearly independent in $V_{\mathbb{Z}[\overline{\mathcal{B}}]}$, hence it is linearly independent in $V_{\Lambda}$.

Step 2. Recall from $\$ 9$ that for each function $f: \mathcal{B} \rightarrow \mathbb{k}$ there is a conformal derivation $\alpha_{f} \in \operatorname{cder} \mathfrak{F}$ such that $N\left(\alpha_{f}, \mathcal{B}\right)=1$ and $\alpha_{f}(0) b=f(b) b$ for every $b \in \mathcal{B}$ (see Lemma $2(\mathrm{a})]$. Let $R=\mathbb{k}\left[\alpha_{f}(n) \mid f \in \mathcal{B}^{*}, n \in \mathbb{Z}_{+}\right]$be the commutative polynomial algebra generated by all $\alpha_{f}(n)$. Lemma 2(b) states that $R$ acts on both $\mathfrak{F}$ and $V_{\Lambda}$ and these actions agree with $\varphi$. 
Fix an element $a \in \mathcal{B}$ and let $f: \mathcal{B} \rightarrow \mathbb{k}$ be defined by $f(b)=\delta_{a, b}$. Denote the corresponding conformal derivation by $a^{\vee}=\alpha_{f} \in \operatorname{cder} \mathfrak{F}$. The algebra $R$ is graded by $\Lambda_{+} \oplus \mathbb{Z}$ by setting wt $a^{\vee}(n)=a, \operatorname{deg} a^{\vee}(n)=-n$.

Our next observation reduces the problem to the following claim.

Claim A. For every homogeneous non-trivial linear combination $x$ of the elements of $\mathcal{T}$ such that wt $x=\lambda$ and $\operatorname{deg} x>\operatorname{deg} w_{\min }(\lambda)=\frac{1}{2}(\lambda \mid \lambda)$, there is $r \in R$, such that $\operatorname{deg} r=\operatorname{deg} w_{\min }(\lambda)-\operatorname{deg} x$ and $r x=c w_{\min }(\lambda)$ for some constant $0 \neq c \in \mathbb{k}$.

Here $w_{\min }(\lambda)$ is given by (14).

Indeed, since $\varphi$ is homogeneous, it is enough to show that $\varphi(x) \neq 0$ for a homogeneous linear combination $x$ of $\mathcal{T}$. If $x=w_{\min }(\lambda)$, then $\varphi(x)=$ $\pm v_{\lambda} \neq 0$ by Proposition 11. Otherwise, using Lemma 2(b) we get $r \varphi(x)=$ $k \varphi\left(w_{\min }(\lambda)\right) \neq 0$, hence $\varphi(x) \neq 0$.

Step 3. Let $w=a_{1}\left(m_{1}\right) \cdots a_{k}\left(m_{k}\right) \in \mathcal{X}^{*}$ be a word in $\mathcal{X}$ and consider a monomial $p=a_{1}^{\vee}\left(n_{1}\right) \cdots a_{k}^{\vee}\left(n_{k}\right) \in R$. Let $G<S_{k}$ be a subgroup of the group of permutations of $k$ elements consisting of permutations that fix the $k$-tuple $\left(a_{1}, \ldots, a_{k}\right)$, and let $H<G$ be the subgroup of $G$ consisting of permutations that in addition fix $\left(n_{1}, \ldots, n_{k}\right)$. We show that Claim $\mathrm{A}$ follows from

Claim B. There exists an element $r_{p} \in R$ such that

$$
r_{p} w=\frac{1}{|H|} \sum_{\sigma \in G} a_{1}\left(m_{1}+n_{\sigma(1)}\right) \cdots a_{k}\left(m_{k}+n_{\sigma(k)}\right) .
$$

Note that every word that appears in the right-hand side of 22 has coefficient 1.

Indeed, suppose Claim B is true. Define an order on $\mathbb{Z}^{k}$ by letting $\left(l_{1}, \ldots, l_{k}\right)>\left(l_{1}^{\prime}, \ldots, l_{k}^{\prime}\right)$ if there is $1 \leqslant j \leqslant k$ such that $l_{i}=l_{i}^{\prime}$ for $j+1 \leqslant i \leqslant k$, but $l_{j}>l_{j}^{\prime}$.

Suppose that $w \in \mathcal{T}$, and let $\left(n_{1}, \ldots, n_{k}\right)=\eta(w) \in \mathbb{Z}^{k}$ be given by (15). Recall that $n_{1} \geqslant n_{2} \geqslant \ldots \geqslant n_{k}$ and if $n_{i}=n_{i+1}$ then $a_{i} \leqslant a_{i+1}$. By Proposition 1 we have $\rho\left(a_{1}\left(m_{1}+n_{1}\right) \cdots a_{k}\left(m_{k}+n_{k}\right)\right)=w_{\min }($ wt $w) \neq 0$. Since $\mathcal{T}$ linearly spans $\mathfrak{F}$, every word of weight wt $w$ and degree less than $\operatorname{deg} w$ is 0 (in other words, Proposition 2 holds). Therefore, if $\left(n_{1}^{\prime}, \ldots, n_{k}^{\prime}\right)>$ $\left(n_{1}, \ldots, n_{k}\right)$ then $\rho\left(a_{1}\left(m_{1}+n_{1}^{\prime}\right) \cdots a_{k}\left(m_{k}+n_{k}^{\prime}\right)\right)=0$.

If $\sigma \in G \backslash H$, then $\left(n_{1}, \ldots, n_{k}\right)<\left(n_{\sigma(1)}, \ldots, n_{\sigma(k)}\right)$. Therefore, (22) implies that

$$
r_{p} \rho(w)=\rho\left(a_{1}\left(m_{1}+n_{1}\right) \cdots a_{k}\left(m_{k}+n_{k}\right)\right) \neq 0 .
$$


This proves Claim A for $x=w \in \mathcal{T}$.

Take now another word $u \in \mathcal{T}$ such that $u \neq w$, wt $u=\operatorname{wt} w, \operatorname{deg} u=$ $\operatorname{deg} w$. Let $\mu \in S_{k}$ be a permutation such that $u=a_{\mu(1)}\left(m_{1}^{\prime}\right) \cdots a_{\mu(k)}\left(m_{k}^{\prime}\right)$. Set $\left(n_{1}^{\prime}, \ldots, n_{k}^{\prime}\right)=\eta(u) \in \mathbb{Z}^{k}$. Suppose that $\left(n_{1}^{\prime}, \ldots, n_{k}^{\prime}\right) \leqslant\left(n_{1}, \ldots, n_{k}\right)$. Then we claim that $r_{p(w)} \rho(u)=0$. Indeed, we have

$$
r_{p(w)} u=\sum_{\sigma \in \mu G \mu^{-1}} a_{\mu(1)}\left(m_{1}^{\prime}+n_{\sigma(1)}\right) \cdots a_{\mu(k)}\left(m_{k}^{\prime}+n_{\sigma(k)}\right) .
$$

This belongs to $\operatorname{Ker} \rho$ if we show that $\left(n_{1}^{\prime}, \ldots, n_{k}^{\prime}\right)<\left(n_{\sigma(1)}, \ldots, n_{\sigma(k)}\right)$ for all $\sigma \in \mu G \mu^{-1}$. If there were an equality in $\left(n_{1}^{\prime}, \ldots, n_{k}^{\prime}\right) \leqslant\left(n_{1}, \ldots, n_{k}\right) \leqslant$ $\left(n_{\sigma(1)}, \ldots, n_{\sigma(k)}\right)$, then $\eta(u)$ would define the same colored partition of $\operatorname{deg} w-\operatorname{deg}_{\min }($ wt $w)$ as does $\eta(w)$, and this contradicts the assumption $u \neq w \in \mathcal{T}$, see $\$$.

Now we can prove Claim A. Let $x \in \mathbb{k}\langle\mathcal{X}\rangle$ be a homogeneous linear combination of words in $\mathcal{T}$. To every word $u=a_{1}\left(m_{1}\right) \cdots a_{k}\left(m_{k}\right) \in \mathcal{T}$ that appears in this combination we correspond the sequence $\left(n_{1}, \ldots, n_{k}\right)=$ $\eta(u) \in \mathbb{Z}^{k}$, given by (15). All these sequences are pairwise different, since the words in $x$ are all of the same weight and degree. Let $w$ be the word that yields the maximal sequence. Take $p=p(w) \in R$ as above, and let $r_{p}$ be as in Claim B. Then the above argument shows that $0 \neq r_{p} w \in \mathbb{k} w_{\min }(\operatorname{wt} w)$ and $r_{p} u=0$ for every other word $u \in \mathcal{T}$ that appears in $x$.

Step 4. It remains to construct for a word $w=a_{1}\left(m_{1}\right) \cdots a_{k}\left(m_{k}\right) \in \mathcal{X}^{*}$ and for a monomial $p=a_{1}^{\vee}\left(n_{1}\right) \cdots a_{k}^{\vee}\left(n_{k}\right) \in R$ an element $r_{p} \in R$ such that (22) holds. Denote the right-hand side of (22) by $S_{p}(w)$.

Let us introduce a partial order on the set of monomials in $R$. Suppose $a_{i}=a_{j}=a$ for some $1 \leqslant i \neq j \leqslant k$, and consider the result of substituting $a^{\vee}\left(n_{i}\right) a^{\vee}\left(n_{j}\right)$ by $a^{\vee}\left(n_{i}+n_{j}\right) a(0)$ in $p$. We write $q \prec p$ if $q$ can be obtained by a number of such substitutions.

Let us now calculate the action of $p$ on $w$. Recall that $R$ acts on $\mathbb{k}\langle\mathcal{X}\rangle$ by derivations, so that $a_{i}(n)^{\vee}\left(a_{j}(m)\right)=\delta_{i, j} a_{j}(m+n)$. For $1 \leqslant j \leqslant k$ denote $\Theta_{j}=\left\{i \mid a_{i}=a_{j}\right\} \subset\{1, \ldots, k\}$. Then

$$
p w=\sum_{i_{1} \in \Theta_{1}, \ldots, i_{k} \in \Theta_{k}} a_{1}\left(m_{1}+\sum_{j=1}^{k} \delta_{i_{j}, 1} n_{j}\right) \cdots a_{k}\left(m_{k}+\sum_{j=1}^{k} \delta_{i_{j}, k} n_{j}\right) .
$$

The crutial observation is that

$$
p w=\sum_{q \preccurlyeq p} c(p, q) S_{q}(w)
$$


for some positive integers $c(p, q)$. Using this, we construct $r_{p}$ by induction on the number $l=l(p)=\#\left\{1 \leqslant j \leqslant k \mid n_{j} \neq 0\right\}$. If $l=0$ or 1 , then $q \preccurlyeq p$ implies $q=p$ and we take $r_{p}=c(p, p)^{-1} p$. Otherwise, set

$$
r_{p}=c(p, p)^{-1}\left(p-\sum_{q \supsetneqq p} c(p, q) r_{q}\right) .
$$

\section{REFERENCES}

1. G. M. Bergman. The Diamond lemma for ring theory. Adv. in Math., 29(2):178-218, 1978.

2. R. E. Borcherds. Vertex algebras, Kac-Moody algebras, and the Monster. Proc. Nat. Acad. Sci. U.S.A., 83(10):3068-3071, 1986.

3. S.-J. Cheng and V. G. Kac. Conformal modules. Asian J. Math., 1(1):181-193, 1997. Erratum: 2(1):153-156, 1998.

4. P. M. Cohn. Universal algebra, volume 6 of Mathematics and its Applications. D. Reidel Publishing Co., Dordrecht, second edition, 1981.

5. C. Dong. Vertex algebras associated with even lattices. J. Algebra, 161(1):245-265, 1993.

6. C. Dong and J. Lepowsky. Generalized vertex algebras and relative vertex operators. Birkhäuser Boston Inc., Boston, MA, 1993.

7. E. Frenkel, V. Kac, A. Radul, and W. Wang. $\mathcal{W}_{1+\infty}$ and $\mathcal{W}\left(\mathfrak{g l}_{N}\right)$ with central charge N. Comm. Math. Phys., 170(2):337-357, 1995.

8. I. B. Frenkel, Y.-Z. Huang, and J. Lepowsky. On axiomatic approaches to vertex operator algebras and modules. Mem. Amer. Math. Soc., 104(494), 1993.

9. I. B. Frenkel, J. Lepowsky, and A. Meurman. Vertex Operator Algebras and the Monster. Academic Press, Boston, MA, 1988.

10. V. G. Kac. Infinite-Dimensional Lie Algebras. Cambridge University Press, Cambridge, third edition, 1990.

11. V. G. Kac. Vertex Algebras for Beginners, volume 10 of University Lecture Series. AMS, Providence, RI, second edition, 1998.

12. J. Lepowsky and R. L. Wilson. A new family of algebras underlying the RogersRamanujan identities and generalizations. Proc. Nat. Acad. Sci. U.S.A., 78(12, part 1):7254-7258, 1981.

13. H. Li. Local systems of vertex operators, vertex superalgebras and modules. J. Pure Appl. Algebra, 109(2):143-195, 1996.

14. H. Li and X. Xu. A characterization of vertex algebras associated to even lattices. J. Algebra, 173(2):253-270, 1995.

15. M. Roitman. On free conformal and vertex algebras. J. Algebra, 217(2):496-527, 1999.

16. M. Roitman. Universal enveloping conformal algebras. Selecta Math. (N.S.), 6(3):319-345, 2000.

17. M. Roitman. Vertex operator realizations of conformal algebras, 2001. To appear. 